\title{
Variations of Hodge Structure Considered as an Exterior Differential System: Old and New Results ${ }^{\star}$
}

\author{
James CARLSON ${ }^{\dagger}$, Mark GREEN ${ }^{\ddagger}$ and Phillip GRIFFITHS $\S$ \\ † Clay Mathematics Institute, United States \\ E-mail: jxxcarlson@mac.com \\ $\ddagger$ University of California, Los Angeles, CA, United States \\ E-mail:mlgucla@gmail.com \\ $\S$ The Institute for Advanced Study, Princeton, NJ, United States \\ E-mail: pg@ias.edu
}

Received April 20, 2009, in final form August 31, 2009; Published online September 11, 2009 doi:10.3842/SIGMA.2009.087

\begin{abstract}
This paper is a survey of the subject of variations of Hodge structure (VHS) considered as exterior differential systems (EDS). We review developments over the last twenty-six years, with an emphasis on some key examples. In the penultimate section we present some new results on the characteristic cohomology of a homogeneous Pfaffian system. In the last section we discuss how the integrability conditions of an EDS affect the expected dimension of an integral submanifold. The paper ends with some speculation on EDS and Hodge conjecture for Calabi-Yau manifolds.
\end{abstract}

Key words: exterior differential systems; variation of Hodge structure, Noether-Lefschetz locus; period domain; integral manifold; Hodge conjecture; Pfaffian system; Chern classes; characteristic cohomology; Cartan-Kähler theorem

2000 Mathematics Subject Classification: 14C30; 58A15

A portion of this paper was presented by the third named author during the Conference on Exterior Differential Systems and Control Theory held at the Mathematical Science Research Institute in Berkeley. This conference was held in honor of Robby Gardner, whose contributions to both exterior differential systems and control theory were of the greatest significance. The authors would like to thank the organizers for putting together the conference and would like to dedicate this paper to the memory of Robby Gardner.

\section{Contents}

1 Introduction $\quad 2$

2 Preliminaries $\quad 5$

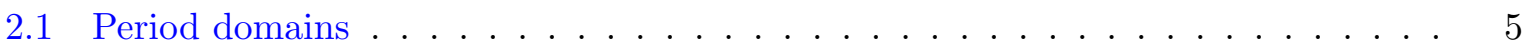

2.2 Exterior differential systems $($ EDS $) \ldots \ldots \ldots \ldots$

3 The exterior differential system associated to a variation of Hodge structure 10 3.1 Elementary examples . . . . . . . . . . . . . . . . . . 11

3.2 A Cartan-Kähler example and a brief guide to some of the literature . . . . . . . 18

3.3 The derived flag of the EDS associated to a VHS . . . . . . . . . . . . . 23

${ }^{\star}$ This paper is a contribution to the Special Issue "Élie Cartan and Differential Geometry". The full collection is available at http://www.emis.de/journals/SIGMA/Cartan.html 
4 Universal cohomology associated to a homogeneous Pfaffian system $\quad 27$

4.1 EDS aspects of homogeneous Pfaffian systems _ . . . . . . . . . . . 27

4.2 Characteristic cohomology of the homogeneous Pfaffian system associated to a VHS 28

5 "Expected" dimension counts for integral manifolds of an EDS

References

\section{Introduction}

Hodge theory provides the basic invariants of a complex algebraic variety. The two central open problems in the subject, the conjectures of Hodge and of Bloch-Beilinson, relate Hodge theory to the geometry/arithmetic of a complex algebraic variety.

The space of all polarized Hodge structures of weight $n$ and with a given sequence $\mathbf{h}=$ $\left(h^{n, 0}, h^{n-1,1}, \ldots, h^{0, n}\right), h^{p, q}=h^{q, p}$, of Hodge numbers forms naturally a homogeneous complex manifold $D_{\mathbf{h}}$, and the moduli space $\mathcal{M}_{\mathbf{h}}$ of equivalence classes of polarized Hodge structures is a quotient of $D_{\mathbf{h}}$ by an arithmetic group acting properly discontinuously. In what we shall call the classical case when the weights $n=1$ or $n=2$ and $h^{2,0}=1, D_{\mathbf{h}}$ is a bounded symmetric domain and $\mathcal{M}_{\mathbf{h}}$ is a quasi-projective variety defined over a number field ${ }^{1}$. In this case the relation between the Hodge theory and geometry/arithmetic of a variety is an extensively developed and deep subject.

In the non-classical, or what we shall refer to as the higher weight, case $^{2}$ the subject is relatively less advanced. The fundamental difference between the classical and higher weight cases is that in the latter case the Hodge structures associated to a family of algebraic varieties satisfy a universal system $I \subset T^{*} D$ of differential equations. In this partly expository paper we will discuss the system $I$ from the perspective of exterior differential systems (EDS's) with the three general objectives:

(i) To summarize some of what is known about $I$ from an EDS perspective.

(ii) To define and discuss the "universal characteristic cohomology" associated to a homogeneous Pfaffian system in the special case of a variation of Hodge structures.

(iii) To discuss and illustrate the question "How must expected dimension counts be modified for integral manifolds of the system $I$ ?"

One overarching objective is this: When one seeks to extend much of the rich classical theory, including the arithmetic aspects and the connections with automorphic forms, the various compactifications of $\mathcal{M}$ and the resulting boundary cohomology, the theory of Shimura varieties, etc., the fact that families of Hodge structures arising from geometry are subject to differential constraints seems to present the major barrier. Perhaps by better understanding the structure of these differential constraints, some insight might be gained on how at least some aspects of the classical theory might be extended. We are especially interested in properties of variations of Hodge structure that are not present in the classical case, as these may help to indicate what needs to be better understood to be able to extend the classical case to higher weight Hodge structures.

In more detail, in Section 2.1 we will review the definitions and establish notations for polarized Hodge structures, period domains and their duals, and the infinitesimal period relation, which is the basic exterior differential system studied in this paper. In Section 2.2 we recall some of the basic definitions and concepts from the theory of exterior differential systems.

\footnotetext{
${ }^{1}$ Henceforth we shall drop reference to the $\mathbf{h}$ on $D$ unless it is needed.

${ }^{2}$ It being understood that when $n=2$ we have $h^{2,0} \geqq 2$.
} 
In Section 3 we discuss the basic exterior differential system whose integral manifolds define variations of Hodge structure. The basic general observation is that the integral elements are given by abelian subalgebras

$$
\mathfrak{a} \subset \mathcal{G}^{-1,1} \subset \mathcal{G}_{\mathbb{C}}
$$

of the complexified Lie algebras of the symmetry group of the period domain. We then go on in Section 3.1 to discuss in some detail two important examples where the EDS given by the infinitesimal period relation may be integrated by elementary methods; both of these have been discussed in the literature and here we shall summarize, in the context of this paper those results. Then in Section 3.2 we shall discuss an example, the first we are aware of in the literature, where the Cartan-Kähler theoretic aspects of exterior differential systems are applied to the particular EDS arising from the infinitesimal period relation. Finally, in Section 3.3 we study the derived flag of the infinitesimal period relation $I$. One result is that if all the Hodge numbers $h^{p, q}$ are non-zero, then the derived flag of $I$ terminates in zero and has length $m$ where $m \leqq \frac{\log n}{\log 2}$, $n$ being the weight of the Hodge structure.

Over the years there have been a number of studies of the EDS given by the infinitesimal period relation. Here we mention $[1,6,7,8,9,10,11]$ and [18]. Section 3 should be considered as an introduction to those works. In particular the paper [18], which builds on and extends the earlier works, contains a definitive account of the bounds on the dimension and rigidity properties of maximal integral elements. At the end of Section 3.3 we shall comment on some interesting questions that arise from [18] and [1] as well as providing a brief guide to the earlier works referred to above.

In Section 4, we first discuss some general aspects of homogeneous Pfaffian systems, including expressing the invariant part of their characteristic cohomology in terms of a Lie algebra cohomology construction ${ }^{3}$. We then turn to the group invariant characteristic cohomology of period domains. Here there is a very nice question

(1.1) Is the invariant part of the characteristic cohmology of a period domain generated by the Chern forms of the Hodge bundles?

In the classical case the answer is positive and may be deduced from what is known in the literature; we will carry this out below.

In the non-classical case when the Pfaffian system $I$ associated to a variation of Hodge structures is non-trivial, new and interesting issues arise. It seems likely that the question will have an affirmative answer; this will be the topic of a separate work ${ }^{4}$.

In this paper we will establish two related results. The first is that we will show that the invariant forms modulo the algebraic ideal generated by $I$ are all of type $(p, p)$. A consequence is that on the complex of invariant forms the Lie algebra cohomology differential $\delta=0$. This is analogous to what happens in the Hermitian symmetric case. However, in the non-classical case there are always more invariant forms than those generated by the Chern forms of the Hodge bundles, and the integrability conditions; i.e. the full differential ideal generated by I must be taken into account. This involves subtle issues in representation theory and, as mentioned above, this story will be reported on separately.

What we will prove here is that the integrability conditions imply topological conditions in the form of new relations among the Chern classes of a VHS. Denoting by $\mathcal{F}^{p}$ the Hodge filtration bundles we show that the Chern forms satisfy

$$
c_{i}\left(\mathcal{F}^{p}\right) c_{j}\left(\mathcal{F}^{n-p}\right)=0 \quad \text { if } \quad i+j>h^{p, n-p} .
$$

\footnotetext{
${ }^{3}$ Here, we recall that the characteristic cohomology of an exterior differential system is the de Rham cohomological construction that leads to cohomology groups that induce ordinary de Rham cohomology on integral manifolds of the EDS. The precise definition is recalled in Section 4 below.

${ }^{4}$ This question has now been answered in the affirmative and the proof will appear in a separate publication.
} 
What we see then is that this new algebro-geometric information has resulted from EDS considerations.

The question (1.1) would have the following algebro-geometric consequence: First, for any discrete group $\Gamma$ acting properly discontinuously on a period domain $D$, the invariant characteristic cohomology $H_{\mathcal{J}}^{*}(D)^{G_{\mathbb{R}}}$ induces characteristic cohomology on the quotient $\Gamma \backslash D$. A global variation of Hodge structure is given by a period mapping

$$
f: S \rightarrow \Gamma \backslash D,
$$

where $S$ is a smooth, quasi-projective algebraic variety. Since (1.2) is an integral manifold of the canonical EDS on $\Gamma \backslash D$, the invariant characteristic cohomology induces ordinary cohomology

$$
f^{*}\left(H_{\mathcal{J}}^{*}(D)^{G_{\mathbb{R}}}\right) \subset H^{*}(S) .
$$

We may think of $H_{\mathcal{J}}^{*}(D)^{G_{\mathbb{R}}}$ as universal characteristic cohomology for the exterior differential system corresponding to the infinitesimal period relation, in that it induces ordinary cohomology on the parameter space for variations of Hodge structures irrespective of the particular group $\Gamma$. We may thus call it the universal characteristic cohomology. A positive answer to the question would first of all imply that the universal characteristic cohomology is generated by the Chern classes of the Hodge bundles over $S$. Although, perhaps not surprising to an algebraic geometer this would be a satisfying result.

In Section 5 we turn to the interesting question

How must one correct expected dimension counts in the presence of differential constraints?

Specifically, given a manifold $A$ and submanifold $B \subset A$, for a "general" submanifold $X \subset A$ where $\operatorname{dim} X+\operatorname{dim} B \geqq \operatorname{dim} A$, we will have

$$
\operatorname{codim}_{B}(X \cap B)=\operatorname{codim}_{A} B
$$

Thus, the RHS of this equation may be thought of as the "expected codimension" of $X \cap B$ in $B$. If $X \cap B$ is non-empty, the actual codimension is no more than the expected codimension.

Suppose now that there is a distribution $W \subset T A$ and $X$ is constrained to have $T X \subset W$. Then how does this affect the expected dimension counts? In case $W$ meets $T B$ transversely, one sees immediately that the "expected codimension" counts decrease. Taking integrability into account gives a further correction. Rather than trying to develop the general theory, in Section 5 we shall discuss one particularly interesting special case.

This case concerns Noether-Lefschetz loci. Here we denote by $W_{I} \subset T D$ the distribution $I^{\perp}$; integral manifolds of $I$ have their tangent spaces lying in $W_{I}$. Given $\zeta \in H_{\mathbb{R}}$, there is a homogeneous sub-period-domain $D_{\zeta} \subset D$, defined as the set of polarized Hodge structures of weight $n=2 m$ where $\zeta \in H^{m, m}$. We have

$$
\operatorname{codim}_{D} D_{\zeta}=h^{(2 m, 0)}+\cdots+h^{(m+1, m-1)},
$$

and the distribution $W_{I}$ on $D$ meets $T D_{\zeta}$ transversely. For a variation of Hodge structure, given by an integral manifold

$$
f: S \rightarrow D
$$

of the canonical system $I$ on $D$, the Noether-Lefschetz locus $S_{\zeta} \subset S$ is given by

$$
S_{\zeta}=f^{-1}\left(D_{\zeta}\right)
$$


In algebro-geometric questions, $\zeta$ is usually taken to be a rational vector, but that will not concern us here. The refined codimension estimate given by $W_{I}$ alone, i.e., without taking integrability conditions into account, is

$$
\operatorname{codim}_{S} S_{\zeta} \leqq h^{m-1, m+1} .
$$

In the case $m=1$, which algebro-geometrically reflects studying codimension one algebraic cycles, the distribution $W_{I}$ does not enter and the estimate is classical, especially in the study of curves on an algebraic surface. In the case $m \geqq 2$, it is non-classical and seems only recently to have been discussed in the literature (cf. $[15,21,19,20]$ ). When the integrability conditions are taken into account, the above codimension estimate is refined to

$$
\operatorname{codim}_{S} S_{\zeta} \leqq h^{m-1, m+1}-\sigma_{\zeta},
$$

where $\sigma_{\zeta}$ is a non-negative quantity constructed from $\zeta$ and the integral element of $I$ given by the tangent space to $f(S)$ at the point in question. Assuming the Hodge conjecture, the above would say that "there are more algebraic cycles than a naïve dimension count would suggest".

In addition to establishing the above inequality, we will show that it is an equality in a significant example, namely, that given by a hypersurface $X \subset \mathbb{P}^{5}$ of degree $d \geqq 6$ and which is general among those containing a 2-plane. This indicates that there is no further general estimate.

We conclude this section by analyzing the case of Calabi-Yau fourfolds, where the quantity $h^{3,1}-\sigma_{\zeta}$ has a particularly nice interpretation, including an interesting arithmetic consequence of the Hodge conjecture.

\section{Preliminaries}

\subsection{Period domains ${ }^{5}$}

Let $H$ be a $\mathbb{Q}$-vector space. A Hodge structure (HS) of weight $n$ is given by any of the following equivalent data:

$$
\begin{aligned}
& \text { A Hodge decomposition } \\
& \left\{\begin{array}{l}
H_{\mathbb{C}}=\underset{p+q=n}{\oplus} H^{p, q} \\
H^{q, p}=\bar{H}^{p, q}
\end{array}\right.
\end{aligned}
$$

(2.2) A Hodge filtration $F^{n} \subset F^{n-1} \subset \cdots \subset F^{0}=H_{\mathbb{C}}$ where for each $p$

$$
F^{p} \oplus \bar{F}^{n-p+1} \stackrel{\sim}{\rightarrow} H_{\mathbb{C}} .
$$

(2.3) A homomorphism of real Lie groups

$$
\varphi: \mathbb{C}^{*} \rightarrow \mathrm{GL}\left(H_{\mathbb{R}}\right)
$$

of weight $n$ in the sense that for $z \in \mathbb{C}^{*}, \lambda \in \mathbb{R}^{*}$

$$
\varphi(\lambda z)=\lambda^{n} \varphi(z) .
$$

The relation between (2.1) and (2.2) is

$$
\left\{\begin{array}{l}
F^{p}=\underset{p^{\prime} \geqq p}{\bigoplus} H^{p^{\prime}, n-p^{\prime}}, \\
H^{p, q}=F^{p} \cap \bar{F}^{q} .
\end{array}\right.
$$

\footnotetext{
${ }^{5}$ The general reference for this section is the book [12].
} 
The relation between $(2.1)$ and (2.3) is

$$
\varphi(z) u=z^{p} \bar{z}^{q} u, \quad u \in H^{p, q} .
$$

This means: The element $\varphi(z) \in \mathrm{GL}\left(H_{\mathbb{C}}\right)$ just given lies in the subgroup $\operatorname{GL}\left(H_{\mathbb{R}}\right)$ of $\mathrm{GL}\left(H_{\mathbb{C}}\right)$ and has weight $n$.

If we restrict $\varphi$ to the maximal compact subgroup $S^{1}=\left\{z \in \mathbb{C}^{*}:|z|=1\right\}$ of $\mathbb{C}^{*}$, then for $z \in S^{1}, u \in H^{p, q}$

$$
\varphi(z) u=z^{p-q} u
$$

and this shows how to recover the Hodge decomposition as the $z^{p-q}$ eigenspace of $\varphi$ restricted to $S^{1}$. The Weil operator is defined by

$$
C=\varphi(\sqrt{-1}) .
$$

We define the Hodge numbers $h^{p, q}:=\operatorname{dim} H^{p, q}$, and we set $f^{p}:=\sum_{p^{1} \geqq p} h^{p^{\prime}, n-p^{\prime}}$.

Since the Hodge filtration point of view will be the dominant one in this paper, we shall denote a Hodge structure by $(H, F)$.

Now let

$$
Q: H \otimes H \rightarrow \mathbb{Q}
$$

be a non-degenerate bilinear form satisfying $Q(u, v)=(-1)^{n} Q(v, u)$ for $u, v \in H$. A Hodge structure $(H, F)$ is polarized by $Q$ if the Hodge-Riemann bilinear relations

$$
\left\{\begin{array}{l}
Q\left(F^{p}, F^{n-p+1}\right)=0, \\
Q(C u, \bar{u})>0 \quad \text { for } \quad 0 \neq u \in H_{\mathbb{C}}
\end{array}\right.
$$

are satisfied. The first of these is equivalent to $F^{n-p+1}=\left(F^{p}\right)^{\perp}$. A polarized Hodge structure (PHS) will be denoted by $(H, Q, F)$.

In the definition (2.3), for a polarized Hodge structure we need to restrict $\varphi$ to $S^{1}$ in order to preserve, and not just scale, the polarization.

Definitions. (i) A period domain $D$ is given by the set of polarized Hodge structures $(H, Q, F)$ with given Hodge numbers $h^{p, q}$. (ii) The compact dual $\check{D}$ is given by all filtrations $F$ with $\operatorname{dim} F^{p}=f^{p}$ and which satisfy the first bilinear relation $F^{n-p+1}=\left(F^{p}\right)^{\perp}$ in $(2.4)$.

We shall denote by $G$ the $\mathbb{Q}$-algebraic group $\operatorname{Aut}(H, Q)$, and by $G_{\mathbb{R}}$ and $G_{\mathbb{C}}$ the corresponding real and complex forms. It is elementary that $G_{\mathbb{R}}$ acts transitively on $D$, and choosing a reference point $F_{0} \in D$ we have

$$
D \cong G_{\mathbb{R}} / V
$$

where $V$ is the compact subgroup of $G_{\mathbb{R}}$ preserving the Hodge decomposition $H_{\mathbb{C}}=\underset{p+q=n}{\oplus} H_{0}^{p, q}$ corresponding to $F_{0}$. In terms of $(2.3)$ we note that

$$
\varphi\left(S^{1}\right) \subset V
$$

in fact, $V$ is the centralizer of the circle $\varphi\left(S^{1}\right)$ in $G_{\mathbb{R}}$.

The complex Lie group $G_{\mathbb{C}}$ acts transitively on the compact dual $\check{D}$, and choosing a reference point $F_{0} \in D$ as above we have

$$
\check{D} \cong G_{\mathbb{C}} / B
$$


where $B \subset G_{\mathbb{C}}$ is a parabolic subgroup. We have

$$
V=G_{\mathbb{R}} \cap B .
$$

The compact dual is a projective algebraic variety defined over $\mathbb{Q}$. In fact we have an obvious inclusion

$$
\check{D} \subset \prod_{p=n}^{\left[\frac{n+1}{2}\right]} \operatorname{Grass}\left(f^{p}, H_{\mathbb{C}}\right)
$$

and we may embed $\check{D}$ in a $\mathbb{P}^{N}$ by means of the Plücker coordinates of the flag subspaces $F^{p} \subset H_{\mathbb{C}}$.

Hodge structures and polarized Hodge structures are functorial with respect to the standard operations in linear algebra. In particular, a Hodge structure $(H, F)$ induces a Hodge structure of weight zero on $\operatorname{End}(H)$ where

$$
\operatorname{End}\left(H_{\mathbb{C}}\right)^{r,-r}=\left\{A \in \operatorname{End}\left(H_{\mathbb{C}}\right): A\left(H^{p, q}\right) \subset H^{p+r, q-r}\right\} .
$$

A polarized Hodge structure $\left(H, Q, F_{0}\right)$ induces a polarized Hodge structure on the Lie algebra $\mathcal{G}$ of $G$, where $\mathcal{G}^{r,-r}$ is given by (2.6) and the polarization is induced by the Cartan-Killing form. For later use, we note that from (2.6) we have

$$
\left[\mathcal{G}^{r,-r}, \mathcal{G}^{s,-s}\right] \subseteq \mathcal{G}^{r+s,-(r+s)} .
$$

If we recall the natural identification

$$
T_{F^{p}} \operatorname{Grass}\left(f^{p}, H_{\mathbb{C}}\right) \cong \operatorname{Hom}\left(F^{p}, H_{\mathbb{C}} / F^{p}\right)
$$

it follows that the Lie algebra of $B$ is

$$
\mathfrak{b}=\underset{r \geqq 0}{\oplus} \mathcal{G}^{r,-r} .
$$

The subalgebra

$$
\mathfrak{p}=\underset{r>0}{\bigoplus} \mathcal{G}^{-r, r}
$$

gives a complement to $\mathfrak{b}$ in $\mathfrak{G}_{\mathbb{C}}$ leading to the natural identification

$$
T_{F} \cdot \check{D} \cong \mathfrak{p} .
$$

By (2.7) the subspace

$$
\mathfrak{G}^{-1,1} \subset \mathfrak{p}
$$

is Ad $B$-invariant and therefore defines a $G_{\mathbb{C} \text {-invariant distribution }}$

$$
W_{I} \subset T \check{D},
$$

and, by orthogonality, a Pfaffian system

$$
I \subset T^{*} \check{D} .
$$

The sub-bundle $I$ restricts to a $G_{\mathbb{R}}$-invariant sub-bundle $I \subset T^{*} D$.

Definition. The Pfaffian system $I$ is called the infinitesimal period relation.

It is this exterior differential system that we shall discuss in this paper.

In the literature the distribution $W_{I} \subset T D$ is frequently referred to as the horizontal subbundle. 


\subsection{Exterior differential systems (EDS $)^{6}$}

Although the subject is usually discussed in the smooth category, here we shall work complexanalytically. A Pfaffian system is given by a holomorphic sub-bundle

$$
I \subset T^{*} M
$$

of the cotangent bundle of a complex manifold. Associated to $I$ is the differential ideal

$$
\mathcal{J} \subset \Omega_{M}^{\bullet}
$$

generated by the holomorphic sections of $I$ together with their exterior derivatives. We shall assume that the values of the sections of $\mathcal{J}$ generate a sub-bundle of $\Lambda^{\bullet} T^{*} M$; i.e., $\mathcal{J}$ is locally free. An integral manifold, or just an "integral", of $I$ is given by a complex manifold $N$ and a holomorphic immersion

$$
f: N \rightarrow M
$$

such that

$$
f^{*}(\mathcal{J})=0
$$

If we denote by

$$
W_{I}=I^{\perp} \subset T M
$$

the holomorphic distribution associated to $I$, the condition (2.9) is equivalent to

$$
f_{*}(T N) \subset W_{I} .
$$

An important invariant associated to a Pfaffian system is its derived flag. The exterior derivative induces a bundle map

$$
\delta: I \rightarrow \Lambda^{2} T^{*} M / I \wedge T^{*} M
$$

and recalling our assumption that $\delta$ has constant rank we set

$$
I_{[1]}=\operatorname{ker} \delta
$$

This is again a Pfaffian system, and continuing in this way leads to the derived flag in $T^{*} M$

$$
I \supset I_{[1]} \supset I_{[2]} \supset \cdots \supset I_{[m]}=I_{[m+1]}=\cdots=I_{[\infty]} .
$$

Here, $I_{[\infty]}$ is the largest integrable or Frobenius subsystem of $I$.

Dually, for the distribution we denote by

$$
W_{I}^{[1]}=W_{I}+\left[W_{I}, W_{I}\right]
$$

the distribution generated by $W_{I}$ and the brackets of sections of $W_{I}$. Continuing in this way we obtain the flag in $T M$ dual to (2.10)

$$
W_{I} \subset W_{I}^{[1]} \subset \cdots \subset W_{I}^{[m]}=W_{I}^{[m+1]}=\cdots=W_{I}^{[\infty]} .
$$

\footnotetext{
${ }^{6}$ General references for this section are books [17] and [2]; especially the former contains essentially all the background needed for this work.
} 
We say that $I$ is bracket generating in case $W_{I}^{[\infty]}=T M$, or equivalently $I^{[\infty]}=(0)$. In this case, by the holomorphic version of Chow's theorem we may connect any two points of $M$ by a chain of holomorphic discs that are integral curves of $W_{I}$.

Two central aspects of the theory of exterior differential systems are $(i)$ regular and ordinary integral elements and the Cartan-Kähler theorem, and (ii) prolongation and involution. For the first, an integral element for $\mathcal{J}$ is given by a linear subspace $E \subset T_{x} M$ such that

$$
\theta_{E}=:\left.\theta\right|_{E}=0
$$

for all $\theta \in \mathcal{J}$. We may think of $E$ as an infinitesimal solution of the EDS. Denote by

$$
\pi: G_{p}(T M) \rightarrow M
$$

the bundle whose fibre $\pi^{-1}(x)=\operatorname{Gr}_{p}\left(T_{x} M\right)$ over $x \in M$ is the Grassmannian of $p$-planes in $T_{x} M$. In $G_{p}(T M)$ there is the complex analytic subvariety

$$
G_{p}(I) \subset G_{p}(T M)
$$

of integral elements defined by the Pfaffian system $I$.

Integral elements are constructed one step at a time by solving linear equations. For an integral element $E \in G_{p}(I)$, we define the polar space

$$
H(E)=\left\{v \in T_{x} M: \operatorname{span}\{v, E\} \text { is an integral element }\right\} .
$$

The equations that define $H(E)$

$$
\langle\theta(x), v \wedge E\rangle=0 \quad \text { for all } \quad \theta \in \mathfrak{J}^{p+1}
$$

are linear in $v$, and we measure their rank by defining

$$
r(E)=\operatorname{codim} H(E)=\operatorname{dim} \mathbb{P}(H(E) / E)
$$

Given $E_{0} \in G_{p}(I)$ we choose a $p$-form $\Omega$ such that $\Omega_{E_{0}} \neq 0$. For $\theta \in \mathcal{J}^{p}$ and $E \in G_{p}(T M)$ near $E_{0}$, for each $\varphi \in \mathcal{J}^{p}$ we write

$$
\theta_{E}=f_{\theta}(E) \Omega_{E}
$$

Then $G_{p}(I)$ is locally defined by the analytic equations $f_{\theta}(E)=0$; we say that $E_{0}$ is regular if $G_{p}(I)$ is smoothly defined by these equations.

Given $E \in G_{p}(I)$ we choose a generic flag

$$
0 \subset E_{1} \subset \cdots \subset E_{p-1} \subset E
$$

and define $c_{i}$ to be the rank of the polar equations of $E_{i}$. A central result is

\section{Cartan's test.}

(i) We have

$$
\operatorname{codim} G_{p}(I) \geqq c_{1}+\cdots+c_{p-1}
$$

(ii) If equality holds, then for $k<p$ each $E_{k}$ is regular. 
By definition we say that $E$ is ordinary if equality holds in Cartan's test. We say that $I$ is involutive near an ordinary integral element.

The Cartan-Kähler existence theorem states that an ordinary integral element is locally tangent to an integral manifold. It goes further to say "how many" local integral manifolds there are. This will be briefly mentioned below.

If $G_{p}(I)$ is a submanifold near $E$, but $E$ is not ordinary one needs to prolong the EDS to be able to use the Cartan-Kähler theorem in order to construct local integral manifolds. To explain this, we first observe that there is a canonical Pfaffian system

$$
J \subset T^{*} G_{p}(T M)
$$

defined by writing points of $G_{p}(T M)$ as $(x, E)$ and setting

$$
J_{(x, E)}=\pi^{*}\left(E^{\perp}\right) .
$$

For any immersion $f: N \rightarrow M$ where $\operatorname{dim} N=p$, there is a canonical lift

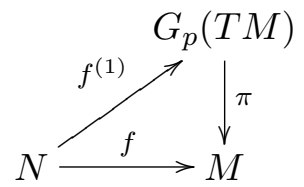

where for $y \in N$ we set $f^{(1)}(y)=\left(f(y), f_{*} T_{y} N\right)$. This lift is an integral manifold of $J$. The converse is true provided the $p$-dimensional integral manifold of $J$ projects to an immersed $p$-dimensional submanifold in $M$.

We define the $1^{\text {st }}$ prolongation $\left(M_{I}^{(1)}, I^{(1)}\right)$ of $(M, I)$ by taking $M_{I}^{(1)}$ to be the smooth locus of $G_{p}(I)$ and defining $I^{(1)}$ to be the restriction of $J$ to $M_{I}^{(1)}$. An integral manifold of $(M, I)$ gives one of $\left(M_{I}^{(1)}, I^{(1)}\right)$; the converse holds in the sense explained above. The Cartan-Kuranishi theorem states that, with some technical assumptions, after a finite number of prolongations an exterior differential system either becomes involutive or else empty (no solutions).

From the above we may infer the following

(2.11) Let $f: N \rightarrow M$ be an integral manifold of a Pfaffian system. If, at a general point $y \in N, f_{*}\left(T_{y} N\right)$ is not an ordinary integral element then one of the following holds:

(A) $f_{*}(T N)$ lies in the singular locus of $G_{p}(I)$; or

(B) the prolongation $f^{(1)}: N \rightarrow M_{I}^{(1)}$ is an integral manifold of $I^{(1)}$.

We may iterate this for the successive prolongations. Since the failure to be involutive means that $f: N \rightarrow M$ satisfies additional differential equations not in $\mathcal{J}$, we have the following

(2.12) Conclusion. If the assumption of (2.11) holds, then $f: N \rightarrow M$ satisfies additional differential equations that are canonically associated to $I$ but are not in the differential ideal $\mathcal{J}$.

\section{The exterior differential system associated to a variation of Hodge structure}

In this section, unless mentioned otherwise we shall work locally. Let $D$ be a period domain and $I \subset T^{*} D$ the infinitesimal period relation with corresponding horizontal distribution $W_{I} \subset T D$. We recall that both $I$ and $W_{I}$ are the restrictions to $D \subset \check{D}$ of similar structures over the compact dual of $D$. 


\subsection{Elementary examples}

Definition. A variation of Hodge structure (VHS) is given by an integral manifold $f: S \rightarrow D$ of $I$.

In the classical case where $D$ is a bounded symmetric domain, it is well known that holomorphic mappings from quasi-projective varieties to quotients $\Gamma \backslash D$ of $D$ by arithmetic groups have strong analytical properties arising from the negative holomorphic sectional curvatures in $T D$. Similar results hold in the general case as a consequence of the horizontality of a VHS. There are also analogous properties that hold for the curvatures of the Hodge bundles. These results are classical; cf. [12] for an exposition and references. In this partly expository paper we will not discuss these curvature properties, but rather we will focus on variations of Hodge structure from an EDS perspective.

We begin with the

(3.1) Basic observation. Integral elements of I are given by abelian subalgebras

$$
\mathfrak{a} \subset \mathcal{G}^{-1,1} .
$$

This result is a consequence of the Maurer-Cartan equation; it is discussed in a more general context at the beginning of Section 4 below (cf. the proof of Proposition 4.2. Here we are using the notations in Section 2.1 above. At a point $F \in D$, the Lie algebra $\mathcal{G}$ has a Hodge structure constructed from $F$, and (3.1) above identifies the space of integral elements of $I$ in $T_{F} D$.

In the theory of EDS, there are two types of exterior differential systems, which may be informally described as follows:

- Those that may be integrated by ODE methods.

- Those that require PDE techniques.

Although the first type may be said to be "elementary", it includes many important EDS's that arise in practice. Roughly speaking, these are the exterior differential systems that, by using ODE's, may be put in a standard local normal form.

We begin by first recalling the contact system and then giving two interesting elementary examples that arise in variations of Hodge structures.

Contact system. Here, $\operatorname{dim} M=2 k+1$ and $I$ is locally generated by a 1 -form $\theta$ with $\theta \wedge$ $(d \theta)^{k} \neq 0$. Then, by using ODE methods, it may be shown that there are local coordinates $\left(x^{1}, \ldots, x^{k}, y, y_{1}, \ldots, y_{k}\right)$ such that $\theta$ may be taken to be given by (using summation convention)

$$
\theta=d y-y_{i} d x^{i}
$$

Local integral manifolds are of dimension $\leqq k$, and those of dimension $k$ on which $d x^{1} \wedge \cdots \wedge d x^{k} \neq$ 0 are graphs

$$
\left(x^{1}, \ldots, x^{k}\right) \rightarrow\left(x^{1}, \ldots, x^{k}, y(x), \partial_{x^{1}} y(x), \ldots, \partial_{x^{k}} y(x)\right) .
$$

Proposition. For weight $n=2$ and Hodge numbers $h^{2,0}=2, h^{1,1}=k, I$ is a contact system [11].

Proof. We shall use the proof to illustrate and mutually relate the two main computational methods that have been used to study the differential system $I$.

The first is using the Hodge structure on $\mathcal{G}$ to identify both the tangent space at $F \in D$ and the fibre $W_{I, F}$ with subspaces of $\mathcal{G}_{\mathbb{C}}$. 
For weight $n=2$ we have for $\mathfrak{p} \cong T_{F} D$ the inclusion

$$
\mathfrak{p} \subset \operatorname{Hom}\left(H^{2,0}, H^{1,1}\right) \oplus \operatorname{Hom}\left(H^{2,0}, H^{0,2}\right) \oplus \operatorname{Hom}\left(H^{1,1}, H^{0,2}\right) .
$$

Using the polarizing form $Q$ gives

$$
\left\{\begin{array}{l}
H^{1,1} \cong \check{H}^{1,1} \\
H^{0,2} \cong \check{H}^{2,0}
\end{array}\right.
$$

Then $\mathfrak{p}$ is given by $A=A_{1} \oplus A_{2} \oplus A_{3}$ in the RHS of (3.2) where

$$
\left\{\begin{array}{l}
A_{2}=-{ }^{t} A_{2} \\
A_{3}={ }^{t} A_{1}
\end{array}\right.
$$

The transpose notation refers to the identification just preceding (3.2). The sub-space $\mathcal{G}^{-1,1}$ is defined by $A_{2}=0$. By (3.1), integral elements are then given by linear subspaces

$$
\mathfrak{a} \subset \operatorname{Hom}\left(H^{2,0}, H^{1,1}\right)
$$

satisfying

$$
A^{t} B=B^{t} A
$$

for $A, B \in \mathfrak{a}$.

The second method is via moving frames. Over an open set in $D$ we choose a holomorphic frame field

$$
\underbrace{\underbrace{e_{1}, \ldots e_{h}}_{F 2}, f_{1}, \ldots, f_{k}}_{F^{1}}, e_{1}^{*}, \ldots, e_{h}^{*}
$$

adapted to the Hodge filtrations and to $Q$ in the sense that

$$
\left\{\begin{array}{l}
Q\left(e_{\alpha}, e_{\alpha}^{*}\right)=1, \\
Q\left(f_{i}, f_{i}\right)=1
\end{array}\right.
$$

and all other inner products are zero. Then, using summation convention,

$$
\left\{\begin{array}{c}
d e_{\alpha}=\theta_{\alpha}^{\beta} e_{\beta}+\theta_{\alpha}^{i} f_{i}+\theta_{\alpha \beta} e_{\beta}^{*}, \\
d f_{i}=\theta_{i}^{\alpha} e_{\alpha}+\theta_{i j} f_{j}+\theta_{i \alpha} e_{\alpha}^{*} .
\end{array}\right.
$$

Denoting $T_{F} D$ by just $T$ and referring to (3.2), (3.3) we have

$$
\begin{aligned}
& \theta_{\alpha}^{i} \in T^{*} \otimes \operatorname{Hom}\left(H^{2,0}, H^{1,1}\right) \leftrightarrow A_{1}, \\
& \theta_{\alpha \beta} \in T^{*} \otimes \operatorname{Hom}\left(H^{2,0}, H^{0,2}\right) \leftrightarrow A_{2} .
\end{aligned}
$$

From $0=d Q\left(e_{\alpha}, e_{\beta}\right)=Q\left(d e_{\alpha}, e_{\beta}\right)+Q\left(e_{\alpha}, d e_{\beta}\right)$ we have

$$
\theta_{\alpha \beta}+\theta_{\beta \alpha}=0
$$

which is the first equation in (3.3). The second equation there is

$$
\theta_{i}^{\alpha}=\theta_{\alpha}^{i}
$$

From the above we see that $I$ is generated by the $h(h-1) / 21$-forms $\theta_{\alpha \beta}$ for $\alpha<\beta$, where $h=h^{2,0}$. When $h=1$, we are in the classical case and $I$ is zero. When $h=2, I$ is generated by a single 1-form $\theta=\theta_{12}$ and is thus a candidate to be a contact system.

To calculate $d \theta$ we use $d\left(d e_{1}\right)=0$, which together with the above formulas give

$$
d \theta \equiv \theta_{1}^{i} \wedge \theta_{2}^{i} \quad \bmod \theta .
$$

Since the 1-forms $\theta_{\alpha}^{i}$ are independent, we see that $\theta \wedge(d \theta)^{k} \neq 0$ as desired. 
The simplest case of this example is when $k=1$. Then $\operatorname{dim} D=3$ and $I$ is locally equivalent to the standard contact system

$$
d y-y^{\prime} d x=0
$$

in $\mathbb{C}^{3}$ with coordinates $\left(x, y, y^{\prime}\right)$.

In general, as noted above the contact system is locally equivalent to the standard one generated by

$$
\theta=d y-y_{i} d x^{i}
$$

in $\mathbb{C}^{2 k+1}$ with coordinates $\left(x^{1}, \ldots, x^{k}, y, y_{1}, \ldots, y_{k}\right)$. Their integral manifolds are graphs

$$
\left(x^{1}, \ldots, x^{k}, y(x), \partial_{x^{1}} y(x), \ldots, \partial_{x^{k}} y(x)\right)
$$

where $y(x)$ is an arbitrary function of $x^{1}, \ldots, x^{k}$. It is of interest to see explicitly how one may construct integral manifolds depending on one arbitrary function of $k$ variables.

For this it is convenient to choose a basis for $H_{\mathbb{C}}$ relative to which

$$
\left.Q=\left(\begin{array}{ccc}
0 & 0 & I \\
0 & -I & 0 \\
I & \underbrace{0}_{h} & \underbrace{0}_{h}
\end{array}\right)\right\} h
$$

Then $F^{2}$ will be spanned by the columns in a matrix

$$
F=(\begin{array}{c}
A \\
B \\
C
\end{array} \underbrace{\} h}_{h}\} h
$$

In an open set we will have $\operatorname{det} C \neq 0$, and it is convenient to normalize to have $C=I$. The equations ${ }^{t} F Q F=0$ are

$$
A+{ }^{t} A={ }^{t} B B \text {. }
$$

The infinitesimal period relation ${ }^{t} F Q d F=0$ is

$$
d A={ }^{t} B d B
$$

Note that adding to this relation its transpose gives the differential of (3.7). Thus

$$
\text { If (3.8) is satisfied and (3.7) is satisfied at one point, then it is satisfied identically. }
$$

We now specialize to the case $h=2$ and write

$$
A=\left(\begin{array}{cc}
A_{11} & A_{12} \\
A_{21} & A_{22}
\end{array}\right), \quad B=\left(\begin{array}{cc}
B_{1} & x^{1} \\
\vdots & \vdots \\
B_{k} & x^{k}
\end{array}\right),
$$

where the $A_{\alpha \beta}, B_{\alpha}, x^{j}$ are independent variables and eventually the $A_{\alpha \beta}$ and $B_{j}$ are to be functions of $x^{1}, \ldots, x^{k}$. The equations (3.8) are

$$
d A_{11}=\Sigma B_{j} d B_{j}=d\left(\frac{\sum B_{j}^{2}}{2}\right) ;
$$


(ii) $\quad d A_{22}=\Sigma x^{j} d x^{j}=d\left(\frac{\sum\left(x^{j}\right)^{2}}{2}\right)$;

(iii) $\quad d A_{12}=B_{j} d x^{j}$;

(iv) $\quad d A_{21}=x^{j} d B_{j}$.

Now $0=d\left(d A_{12}\right)=\Sigma d B_{j} \wedge d x^{2}$ or

$$
B_{j}(x)=\partial_{x^{j}} B(x),
$$

where $B(x)$ is an arbitrary function of $x^{1}, \ldots, x^{k}$.

This then leads to the construction of integral manifolds, here constructed by ODE methods. As previously noted, in general, PDE methods - the Cartan-Kähler theorem - are required.

In the above example the $1^{\text {st }}$ derived system $I_{[1]}=(0)$. Below we shall give an example, usually referred to as the mirror quintic, that shows a different phenomenon. In general we have

$$
d \theta_{\alpha \beta} \equiv \theta_{\alpha}^{i} \wedge \theta_{\beta}^{i} \bmod \operatorname{span}\left\{\theta_{\alpha \beta}{ }^{\prime} \mathrm{s}\right\} .
$$

We may then think of $I$ as a sort of multi-contact system (cf. [18]). Note that for each $\alpha<\beta$

$$
\theta_{\alpha \beta} \wedge\left(d \theta_{\alpha \beta}\right)^{k} \neq 0
$$

and

$$
\bigwedge_{\alpha<\beta}\left(\theta_{\alpha \beta} \wedge\left(d \theta_{\alpha \beta}\right)^{k}\right) \neq 0
$$

Integral elements are spanned by matrices $A_{\alpha \lambda}^{i}$ satisfying (using summation convention)

$$
A_{\alpha \lambda}^{i} A_{\beta \mu}^{i}=A_{\alpha \mu}^{i} A_{\beta \lambda}^{i}
$$

corresponding to subspaces $\operatorname{span}\left\{A_{\alpha \lambda}^{i}\right\} \subset \operatorname{Hom}\left(H^{2,0}, H^{1,1}\right)$ on which the RHS of (3.10) vanishes.

When $h=h^{2,0}=2$, the maximal abelian subalgebras (3.4) have dimension $k=h^{1,1}$ and correspond to Lagrangian subspaces in the symplectic vector space $\mathcal{G}^{-1,1}$. In general, the multicontact nature of $I$ suggests a bound of the sort

$$
\operatorname{dim} \mathfrak{a} \leqq \frac{1}{2}\left(h^{2,0} h^{1,1}\right) .
$$

This is in fact proved in [6], where it is also shown that the bound is sharp for $h^{1,1}$ even. When $h^{1,1}$ is odd, it is shown there that the sharp bound is $\frac{1}{2} h^{2,0}\left(h^{1,1}-1\right)+1$. Because it illustrates yet another method for estimating the dimension of, and actually constructing, integral elements we shall give a proof of this result in the cases $h^{2,0}=2$ and $h^{2,0}=3$. By (3.5), we are looking for a linear subspace

$$
E \subset \operatorname{Hom}\left(H^{2,0}, H^{1,1}\right)
$$

with a basis $A_{1}, \ldots, A_{r}$ satisfying

$$
A_{i}{ }^{t} A_{j}=A_{j}{ }^{t} A_{i}
$$

for all $i, j$. A key fact is

$$
A_{i}(v)=0 \Rightarrow Q\left(A_{j}(v), \text { Image } A_{i}\right)=0
$$

for all $j$. 


\section{Case $h^{2,0}=2$}

We want to show that $r \geqq k+1$ is impossible. Assuming that $r \geqq k+1$ then some $A \in E$ drops rank. With suitable labelling, for some $0 \neq v \in H^{2,0}$ we will have $A_{k+1}(v)=0$. By the above, this gives

$$
A_{i}(v) \in \operatorname{Im}\left(A_{k+1}\right)^{\perp}
$$

for $1 \leqq i \leqq k$. Remembering that $h^{1,1}=k$, we have $\operatorname{dim} \operatorname{Im}\left(A_{k+1}\right)^{\perp} \leqq k-1$ so that $A_{1}(v), \ldots, A_{k}(v)$ are linearly dependent. Relabelling, we may assume that $A_{k}(v)=0$. Now

$$
\operatorname{dim}\left(\operatorname{Im} A_{k}+\operatorname{Im} A_{k+1}\right) \geqq 2,
$$

since otherwise some linear combination of $A_{k}$ and $A_{k+1}$ is zero. Then

$$
A_{i}(v)=\left(\operatorname{Im} A_{k}+\operatorname{Im} A_{k+1}\right)^{\perp}
$$

for $i=1, \ldots, k-1$ forces a linear dependence on $A_{1}(v), \ldots, A_{k-1}(v)$. Proceeding by downward induction gives a contradiction to the assumption $r \geqq k+1$.

\section{Case $h^{2,0}=3$}

Since $r \geqq k-1$ we have $A(v)=0$ for some $0 \neq v \in H^{2,0}, A \in E$. Choosing a basis $A_{1}, \ldots, A_{m}$ for the kernel of the map $v \rightarrow A(v)$, we let

$$
s=\operatorname{dim}\left\{\operatorname{Im} A_{1}+\cdots+\operatorname{Im} A_{m}\right\} .
$$

This gives an injective map

$$
H^{2,0} / \mathbb{C} v \rightarrow \mathbb{C}^{s} \subset \mathbb{C}^{k}
$$

By the previous case, $m \leqq k$. Clearly $m \leqq 2 s$ since $\operatorname{dim}\left(\operatorname{Hom} H^{2,0} / \mathbb{C} v, \mathbb{C}^{k}\right)=2 s$. Thus $m \leqq \min (2 s, k)$. Since for all $A \in E$

$$
A(v) \in\left(\operatorname{Im} A_{1}+\cdots+\operatorname{Im} A_{m}\right)^{\perp} \cong \mathbb{C}^{k-s}
$$

we have $m \geqq r-(k-s)$. Thus $\min (2 s, k) \geqq r-(k-s)$ which gives

$$
k-s+\min (2 s, k) \geqq r
$$

or $r \leqq 3 k / 2$ as desired.

When $k=2 s$ this bound is sharp. To see this, take a subspace $\mathcal{U} \subset H^{1,1} \cong \mathbb{C}^{2 s}$ such that $\left.Q\right|_{u}=0$. Let $e_{1}, e_{2}, e_{3} \in H^{2,0}$ be a basis, and take linearly independent $A_{1}, \ldots, A_{k}$ satisfying

$$
e_{1} \rightarrow 0 \quad \text { and } \quad e_{2}, e_{3} \rightarrow \mathcal{U}
$$

Then take linearly independent $B_{1}, \ldots, B_{s}$ satisfying

$$
e_{1} \rightarrow \mathcal{U} \quad \text { and } \quad e_{2}, e_{3} \rightarrow 0
$$

It follows that $E=\operatorname{span}\left\{A_{1}, \ldots, A_{k}, B_{1}, \ldots, B_{s}\right\}$ is an integral element of dimension $k+s=$ $3 k / 2$. 
Example. We take weight $n=3$ and Hodge numbers $h^{3,0}=h^{2,1}=1$. We will show that in this case $\operatorname{dim} D=4$ and $I$ is locally equivalent to the Pfaffian system

$$
\left\{\begin{array}{c}
d y-y^{\prime} d x=0 \\
d y^{\prime}-y^{\prime \prime} d x=0
\end{array}\right.
$$

in $\mathbb{C}^{4}$ with coordinates $\left(x, y, y^{\prime}, y^{\prime \prime}\right)$. Since $d\left(d y-y^{\prime} d x\right)=-d y^{\prime} \wedge d x=-\left(d y^{\prime}-y^{\prime \prime} d x\right) \wedge d x$, the $1^{\text {st }}$ derived system of (3.11) has rank one. In fact, (3.11) is just the $1^{\text {st }}$ prolongation of the contact system (3.6).

Anticipating the later discussion of the period domain $D$ for general weight $n=3$ Hodge structures with $h^{3,0}=1$ and $h^{2,1}=h$, locally over an open set $\mathcal{U}$ we consider a holomorphic frame field

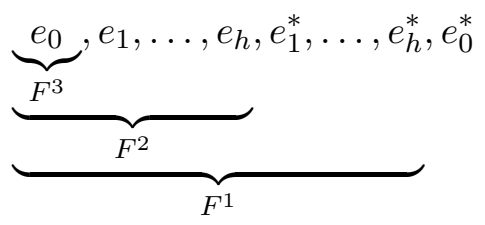

relative to which

$$
\left\{\begin{array}{l}
Q\left(e_{0}, e_{0}^{*}\right)=1=-Q\left(e_{0}^{*}, e_{0}\right), \\
Q\left(e_{\alpha}, e_{\alpha}^{*}\right)=1=-Q\left(e_{\alpha}^{*}, e_{\alpha}\right)
\end{array}\right.
$$

and all other pairings are zero. We observe that

The system $I$ is equivalent to each of the following

$$
\left\{\begin{array}{l}
d e_{0} \equiv 0 \quad \bmod F^{2}, \\
Q\left(d e_{0}, F^{2}\right)=0
\end{array}\right.
$$

the equivalence resulting from $F^{2}=\left(F^{2}\right)^{\perp}$.

We set, again using summation convention,

$$
\begin{cases}d e_{0} \equiv \theta^{\alpha} e_{\alpha}+\theta_{\alpha} e_{\alpha}^{*}+\theta e_{0}^{*} & \bmod F^{3}, \\ d e_{\alpha} \equiv \theta_{\alpha \beta} e_{\beta}^{*}+\theta_{\beta}^{*} e_{0}^{*} & \bmod F^{2} .\end{cases}
$$

Using these equations and the exterior derivatives of (3.12) we have

$$
\left\{\begin{array}{l}
\theta_{\alpha \beta}=\theta_{\beta \alpha} \\
\theta_{\alpha}^{*}=\theta_{\alpha}
\end{array}\right.
$$

We may then conclude

(3.14) The 1-forms $\theta, \theta^{\alpha}, \theta_{\alpha}, \theta_{\alpha \beta}$ for $\alpha \leqq \beta$ are semi-basic for the fibering $G_{\mathbb{C}} \rightarrow \check{D}$, and over $\mathcal{U}$ give bases for the cotangent spaces.

In terms of the Lie algebra description of the tangent space we have

$$
\left\{\begin{array}{l}
\theta^{\alpha}, \theta_{\alpha \beta} \leftrightarrow \mathcal{G}^{-1,1} \\
\theta_{\alpha} \leftrightarrow \mathcal{G}^{-2,2} \\
\theta \leftrightarrow \mathcal{G}^{-3,3}
\end{array}\right.
$$


From the exterior derivatives of (3.13) we obtain

$$
\left\{\begin{array}{l}
d \theta=2 \theta^{\alpha} \wedge \theta_{\alpha}, \\
d \theta_{\beta}=\theta^{\alpha} \wedge \theta_{\alpha \beta} .
\end{array}\right.
$$

From the Lie algebra description of the tangent space, it follows that $I$ is generated by the Pfaffian equations

$$
\left\{\begin{array}{c}
\theta=0 \\
\theta_{\alpha}=0 .
\end{array}\right.
$$

From this we see that the $1^{\text {st }}$ derived system $I_{[1]}$ is generated by $\theta$, and the $2^{\text {nd }}$ derived system $I_{[2]}=0$.

When $h=1$ we are on a 4-dimensional manifold with a rank 2 Pfaffian system $I$ and where $\operatorname{rank} I_{[1]}=1, I_{[2]}=0$. It is well known (Engel's theorem) and elementary [17] and [2, Chapter 2] that such a system is locally equivalent to (3.11).

We now return to the general case when $h^{3,0}=1$ and $h^{2,1}=h$. Because of their importance in algebraic geometry, we shall be interested in integral manifolds $S \subset D$ where the map

$$
T S \rightarrow \operatorname{Hom}\left(H^{3,0}, H^{2,1}\right)
$$

is an isomorphism. We shall call these integral manifolds of Calabi-Yau type.

(3.17) Proposition (cf. [3]). The EDS for integral manifolds of Calabi-Yau type is canonically the $1^{\text {st }}$ prolongation of a contact system.

Thus, locally these integral manifolds depend on one arbitrary function of $h$ variables.

Proof. We set $P=\mathbb{P} H_{\mathbb{C}} \cong \mathbb{P}^{2 h+1}$. Denoting points of $P$ by homogeneous coordinate vectors $[z]=\left[z_{0}, \ldots, z_{2 h+1}\right]$, we consider on $H_{\mathbb{C}}$ the 1 -form

$$
\theta=Q(d z, z) \text {. }
$$

Rescaling locally by $z \rightarrow f z$ where $f$ is a non-vanishing holomorphic function we have

$$
\theta \rightarrow f^{2} \theta .
$$

It follows that $\theta$ induces a 1 -form, defined up to scaling, on $P$. Choosing coordinates so that

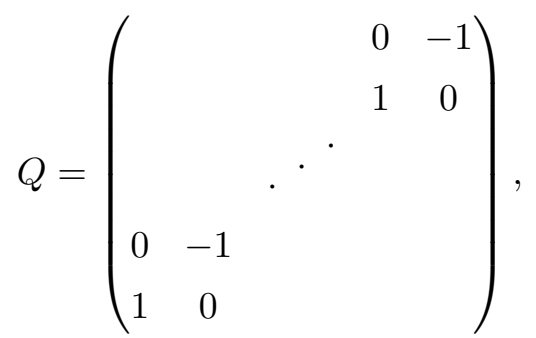

in the standard coordinate system on $P$ where $z_{0}=1$ we have

$$
\begin{aligned}
& \theta=d z_{1}+\sum_{j=1}^{m} z_{2 j} d z_{2 j+1}-z_{2 j+1} d z_{2 j} \\
& d \theta=2 \sum_{j=1}^{m} d z_{2 j} \wedge d z_{2 j+1}
\end{aligned}
$$

from which it follows that $\theta$ induces a contact structure on $P$. 
Now let $S \subset D$ be an integral manifold of Calabi-Yau type and consider the diagram

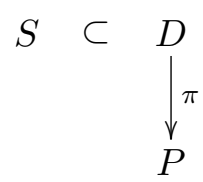

where $\pi(F)=F^{3}$ viewed as a line in $H_{\mathbb{C}}$. Choose local coordinates $s_{1}, \ldots, s_{m}$ on $S$ and write $\left.\pi\right|_{S}$ as

$$
s \rightarrow[z(s)] .
$$

Letting $F_{s}^{p} \subset H_{\mathbb{C}}$ denote the subspace corresponding to $s \in S$, the condition $d F_{s}^{3} \subset F_{s}^{2}=\left(F_{s}^{2}\right)^{\perp}$ gives

$$
\left.\pi^{*} \theta\right|_{S}=Q(d z(s), z(s))=0
$$

i.e., $\pi(S)$ is an integral manifold of the canonical contact system on $P$. In terms of $z(s)$, the integral manifold $S$ is given by

$$
\left\{\begin{array}{l}
F_{s}^{3}=[z(s)], \\
F_{s}^{2}=\operatorname{span}\left\{z(s), \partial_{s_{1}} z(s), \ldots, \partial_{s_{n}} z(s)\right\} \in F(1, h),
\end{array}\right.
$$

where $F(1, h)$ is the manifold of flags $F^{3} \subset F^{2} \subset H_{\mathbb{C}}$ where $\operatorname{dim} F^{3}=1, \operatorname{dim} F^{2}=h+1$, and where we have used the obvious inclusion $\check{D} \subset F(1, h)$.

This process may locally be reversed. Given an $h$-dimensional integral manifold $s \rightarrow[z(s)]$ of the canonical Pfaffian system on $P$, we may define an integral manifold of $I$ by (3.18).

Finally, referring to (3.16) we see that what we denoted by $\theta$ there corresponds to the $\theta$ just above. From equation (3.15) we may infer that $I$ is in fact locally just the $1^{\text {st }}$ prolongation of the canonical contact system on $P$.

Remark. In terms of an arbitrary function $g\left(s_{1}, \ldots s_{h}\right)$ of $h$ variables, in the standard coordinate system the above integral manifold of the contact system is

$$
\left(s_{1}, \ldots, s_{n}\right) \rightarrow\left(g(s), \partial_{s_{1}} g(s), s_{1}, \ldots \partial_{s_{n}} g(s), s_{n}\right) .
$$

It follows that $S \subset D$ is given parametrically in terms of $g(s)$ and its first and second derivatives.

\subsection{A Cartan-Kähler example and a brief guide to some of the literature}

Example. We shall now discuss how the Cartan-Kähler theory applies to the case of weight $n=2, h^{2,0}=3$ and $h^{1,1}=h$. This is the first "non-elementary" case.

The following is a purely linear algebra discussion dealing with the data

- Complex vector spaces $P, R$ of dimension $3, h$ respectively and where $R$ has a nondegenerate symmetric form giving an identification $R \cong \check{R}$.

- $\operatorname{In} \operatorname{Hom}(P, R) \cong \check{P} \otimes R$ we are looking for an abelian subspace $E$, that is a linear subspace of $\check{P} \otimes R$ such that

$$
{ }^{t} A B-{ }^{t} B A=0
$$

for all $A, B \in E$. Here, the transpose is relative to the identification $R \cong \check{R}$. 
We seek $h$-dimensional abelian subspaces $E$ that are in general position relative to the tensorproduct structure in the sense that for a general $u \in P$ the composite map

$$
\begin{array}{cc}
E \rightarrow & \operatorname{Hom}(u, R) \rightarrow R \\
& \\
& \operatorname{Hom}(P, R)
\end{array}
$$

is an isomorphism. With this assumption we shall be able to establish that general integral elements that satisfy it are ordinary in the EDS sense.

It is convenient to choose a basis $e_{i}$ for $P$ and an orthonormal basis $e_{\alpha}$ for $R$ so that

$$
e_{i \alpha}=: \check{e}_{\alpha} \otimes e_{i}
$$

gives a basis for $\operatorname{Hom}(P, R)$. Denote by

$$
\theta_{i}^{\alpha} \in \operatorname{Hom}(P, R)^{\vee}
$$

the dual basis. The notation has been chosen to line up with the "moving frame" notations above. Using the summation convention and setting

$$
\Omega_{i j}=\theta_{i}^{\alpha} \wedge \theta_{j}^{\alpha}=-\Omega_{j i},
$$

abelian subspaces are given by linear subspaces $E$ on which the restriction

$$
\left.\Omega_{i j}\right|_{E}=0 .
$$

We may assume without loss of generality that $E$ is of dimension $h$ and that the condition of general position is satisfied for $u=e_{i}, i=1,2,3$. Setting $\Theta_{i}=\wedge_{\alpha} \theta_{i}^{\alpha}$, we have

$$
\left.\Theta_{i}\right|_{E} \neq 0 .
$$

Then $E$ is defined by linear equations

$$
\begin{cases}\theta_{2}^{\alpha}=A_{\beta}^{\alpha} \theta_{1}^{\beta}, & \operatorname{det}\left\|A_{\beta}^{\alpha}\right\| \neq 0, \\ \theta_{3}^{\alpha}=B_{\beta}^{\alpha} \theta_{1}^{\beta}, & \operatorname{det}\left\|B_{\beta}^{\alpha}\right\| \neq 0 .\end{cases}
$$

The equations (3.19) for $\Omega_{12}$ and $\Omega_{13}$ are (Cartan's lemma)

$$
\left\{\begin{array}{l}
A={ }^{t} A, \\
B={ }^{t} B .
\end{array}\right.
$$

The equation (3.19) for $\Omega_{23}$ is

$$
\left[A, B^{-1}\right]=0 .
$$

In fact, if $\theta_{1}^{\alpha}=C_{\beta}^{\alpha} \theta_{3}^{\beta}$ on $E$, then $A=C B$ and $C={ }^{t} C$ gives (3.21).

(3.22) Proposition. The dimension of the space of generic h-dimensional abelian subspaces is $h(h+3) / 2$.

Proof. The group operating on $\operatorname{Hom}(P, R)$ is $\mathrm{GL}(P) \times O(R)$; this group preserves the set of equations (3.19) that define the abelian subspaces of $\operatorname{Hom}(P, R)$. For elements $I \times T$ in this group, the action on the matrices $A$ and $B$ above is given by

$$
\left\{\begin{array}{l}
A \rightarrow{ }^{t} T A T \\
B \rightarrow{ }^{t} T B T .
\end{array}\right.
$$


For $A$ generic we may find $T$ so that $A=\operatorname{diag}\left(\lambda_{1}, \ldots, \lambda_{h}\right)$ where the $\lambda_{\alpha}$ are non-zero and distinct. The condition (3.21) then gives that $B=\operatorname{diag}\left(\mu_{1}, \ldots, \mu_{h}\right)$. We thus have as a basis for $E$

$$
v_{\alpha}=e_{1 \alpha}+\lambda_{\alpha} e_{2 \alpha}+\mu_{\alpha} e_{3 \alpha}
$$

where $1 \leqq \alpha \leqq h$.

(3.23) Proposition. The equations that define the polar space $H(E)$ have rank $2 h$. Thus $E$ is a maximal integral element.

Proof. For $v=C^{\alpha} e_{1 \alpha}+D^{\alpha} e_{2 \alpha}+E^{\alpha} e_{3 \alpha}$ the equations that express the condition that $\operatorname{span}\{E, v\}$ be an integral element are

$$
\Omega_{i j}\left(v, v_{\beta}\right)=0 .
$$

These compute out to be, with no summation on repeated indices,

$$
\left\{\begin{array}{l}
D^{\beta}=\lambda_{\beta} C^{\beta}, \\
E^{\beta}=\mu_{\beta} C^{\beta}, \\
\mu_{\beta} D^{\beta}=\lambda_{\beta} E^{\beta} .
\end{array}\right.
$$

These are compatible and have rank $2 h$. For any solution, we replace $v$ by $\tilde{v}=v-C^{\alpha} v_{\alpha}$ (summation here), and then $\tilde{v}=0$ by the above equations when all $C^{\alpha}=0$.

(3.24) Proposition. A general abelian subspace $E$ that is in general position with respect to the tensor product structure is an ordinary integral element.

Proof. In order to not have the notation obscure the basic idea, we shall illustrate the argument in the case $h=2$. A general line $E_{1}$ in $E$ is spanned by $w=\rho^{\alpha} v_{\alpha}$ (summation convention). The polar equations for $v$ as above are $\Omega_{i j}(w, v)=0$ for $i<j$. These are

$$
\left\{\begin{array}{l}
\rho^{1}\left(D^{1}-\lambda_{1} C^{1}\right)+\rho^{2}\left(D^{2}-\lambda_{2} C^{2}\right)=0, \\
\rho^{1}\left(E^{1}-\mu_{1} C^{1}\right)+\rho^{2}\left(E^{2}-\mu_{2} C^{2}\right)=0, \\
\rho^{1}\left(\lambda_{1} E^{1}-\mu_{1} D^{1}\right)+\rho^{2}\left(\lambda_{2} E^{2}-\mu_{2} D^{2}\right)=0 .
\end{array}\right.
$$

For a general choice of $\rho^{\alpha}, \lambda_{\alpha}, \mu_{\alpha}$ these are independent. Hence, in the notation of Section 2.2 we have

$$
c_{0}=0, \quad c_{1}=3 .
$$

On the other hand, the 2-dimensional abelian subspaces in $\operatorname{Hom}(P, R) \cong \mathbb{C}^{6}$ is a smooth, 5dimensional subvariety in the 8-dimensional Grassmannian $\operatorname{Gr}(2,6)$. Therefore the codimension of $E$ in $\operatorname{Gr}(2,6)$ is $3=8-5$. Consequently, Cartan's test is satisfied.

In [10] it is shown how to integrate the above system, with the result

(3.25) Integral manifolds of the above system are parametrized by generating functions $f_{1}, f_{2}$ subject to the PDE system

$$
\left[H_{f_{1}}, H_{f_{2}}\right]=0 \text {, }
$$

where $H_{f}$ is the Hessian matrix of $f$. 


\section{A brief guide to some of the literature}

Building on earlier works, in [18] a number of results are proved:

(i) Under rather general assumptions of the type

- the weight $n=2 m+1$ is odd, $h^{m+1, m}>2$ and all other $h^{p, q}>1$,

- the weight $n=2 m$ is even, $h^{m, m}>4, h^{m+2, m-2}>2$ and all other $h^{p, q}>1$

a bound on the dimension of integral elements is obtained, and it is shown that there is a unique integral element $E$ that attains this bound.

(ii) There is a unique germ of integral manifold whose tangent space is $E$.

The following interesting question arises:

Is $E$ an ordinary integral element?

This is a question of computing the ranks of the polar equations of a generic flag in $E$. Setting $\operatorname{dim} E=p$ and $\operatorname{dim} D=N$, since $E$ is unique we have at $E$

$$
\operatorname{codim} G_{p}(I)=\operatorname{dim} \operatorname{Grass}(p, N)=p(N-p)
$$

and Cartan's test is whether the inequality

$$
p(N-p) \geqq c_{1}+\cdots+c_{p-1}
$$

is an equality. Suppose that equality does hold, so that $E$ is ordinary and the Cartan-Kähler theorem applies. As explained in [17] and [2] there are Cartan characters $s_{0}, s_{1}, \ldots, s_{p}$ expressed in terms of the $c_{i}$ such that local integral manifolds of $I$ depend on $s_{p}$ arbitrary functions of $p$-variables, $s_{p-1}$ arbitrary functions of $(p-1)$-variables, $\ldots, s_{0}$ arbitrary functions of 0 -variables (i.e., constants). Mayer's result would then follow by showing that equality holds in Cartan's test and

$$
s_{p}=\cdots=s_{1}=0, \quad s_{0}=1 .
$$

In fact, if $E$ is ordinary then the Cartan characters must be given in this way. Another interesting question is

Do the Mayer integral manifolds arise from algebraic geometry?

Since there is a unique one of these through each point of $D$, one knows that in the non-classical case a general Mayer integral manifold does not arise from geometry. In [9] it is shown that in weight two with $h^{1,1}$ even some maximal integral manifolds are realized geometrically.

Among the works that preceded [18], and in some cases led up to it are:

[11]: In this paper it was recognized that the weight two horizontal distribution is a generalization of the contact distribution.

[7]: Here it is shown that most hypersurface variations are maximal, i.e. their tangent space at a point is not contained in a larger integral element. These integral elements are not of maximal dimension in the sense of [18].

[8]: This paper gives the results in weight two that were extended to the general case in [18]. 
We conclude this section by discussing the recent work [1].

One may think of an integral element $E$ at $F \in D$ as giving an action of Sym $E$ on $\oplus F^{p} / F^{p+1}$ that is compatible with $Q$; i.e., the action preserves the pairings $Q: F^{p} / F^{p+1} \otimes F^{n-p} / F^{n-p+1} \rightarrow \mathbb{C}$. Especially if $\operatorname{dim} E$ is not small, such an action will have many algebraic invariants. Experience suggests that those that arise from geometry will have a special structure.

One such relates to the notion of symmetrizers due to Donagi (cf. [13] and [7]) and used in [1]. Given vector spaces $A, B, C$ and a bilinear map

$$
\Phi: A \otimes B \rightarrow C
$$

one defines

$$
\operatorname{Sym} \Phi=\left\{\Psi \in \operatorname{Hom}(A, B): \Phi\left(a, \Psi\left(a^{\prime}\right)\right)=\Phi\left(a^{\prime}, \Psi(a)\right) \text { for } a, a^{\prime} \in A\right\} .
$$

One may consider the Sym's of the various maps

$$
\mathrm{Sym}^{k} E \otimes H^{p, n-p} \rightarrow H^{p-k, n-p+k}
$$

arising above. In [1] these are studied when $\operatorname{dim} E$ and the $h^{p, n-p}$ are the same as for smooth hypersurfaces $X \subset \mathbb{P}^{n+1}$ where $n \geqq 3$ and $\operatorname{deg} X \geqq n+3$, and where the special structure arising from Macauley's theorem is satisfied. It is shown that a particular Sym is generically zero, but is non-zero in the geometric case. Thus the integral elements arising from hypersurface deformations, which are known to be maximal, satisfy non-trivial algebraic conditions and are thus non-generic.

We shall give a brief discussion of proofs of these results, based on the paper [14], in the case $n=2$. For this we use the notations from the proof of Theorem 5.10 below. For smooth surfaces in $\mathbb{P}^{3}$ the analogous identification to $(5.13)$ is

$$
H^{2-p, p} \cong V^{(p+1) d-4} / J_{(p+1) d-4}
$$

We also have an identification

$$
E \cong V^{d} / J_{d}
$$

Using $E \subseteq \operatorname{Hom}\left(H^{2,0}, H^{1,1}\right)$ there is a map

$$
\operatorname{Hom}\left(H^{2,0}, E\right) \rightarrow \operatorname{Hom}\left(\stackrel{2}{\otimes} H^{2,0}, H^{1,1}\right) .
$$

From the above identifications, we have

$$
V^{4} / J_{4} \subseteq \operatorname{Hom}\left(H^{2,0}, E\right) \cong \operatorname{Hom}\left(V^{d-4}, V^{2 d-4} / J_{2 d-4}\right)
$$

and under the above map $V^{4} / J_{4}$ lands in $\operatorname{Hom}\left(\operatorname{Sym}^{2} H^{2,0}, H^{1,1}\right)$. Put another way

$$
V^{4} / J_{4} \subseteq \operatorname{ker}\left\{\operatorname{Hom}\left(H^{2,0}, E\right) \rightarrow \operatorname{Hom}\left(\wedge^{2} H^{2,0}, E\right)\right\}
$$

It may be seen that for $d \geqq 5$ and for $E$ a general integral element, this kernel is non-zero. This is illustrative of the non-genericity results in [1].

If follows from [14] that for $d \geqq 5$

$$
V^{4} \rightarrow \check{H}^{2,0} \otimes E \rightarrow \wedge^{2} \check{H}^{2,0} \otimes H^{1,1}
$$

is exact at the middle term. Thus one has 
Integrability. The composition

$$
H^{2,0} \rightarrow \check{E} \otimes H^{1,1} \rightarrow \wedge^{2} \check{E} \otimes H^{0,2}
$$

is zero.

Non-generic condition. The kernel

$$
\operatorname{ker}\left\{\check{H}^{2,0} \otimes E \rightarrow \wedge^{2} \check{H}^{2,0} \otimes H^{1,1}\right\}
$$

is non-zero, and as a vector space is isomorphic to $V^{4}$.

One may next proceed, for $d \geqq 8$, to look at

$$
V^{d-8} \rightarrow \operatorname{Hom}\left(V^{4}, H^{2,0}\right) \rightarrow \operatorname{Hom}\left(\wedge^{2} V^{4}, E\right)
$$

and by [14] this will be exact at the middle term of $d \geqq 9$. This process continues and leads to a large number of non-linear constraints on the integral element arising from variations of a smooth surface of degree $d \geqq 5$.

Additional constraints, of a different character, arise as follows. Recall that polynomials $f_{0}$, $f_{1}, f_{2}, f_{3}$, where $f_{i} \in V^{d}$, form a regular sequence if $\operatorname{Var}\left(f_{0}, f_{1}, f_{2}, f_{3}\right)=\emptyset$ in $\mathbb{P}^{3}$. A special case is when

$$
f_{i}(x)=F_{x_{i}}(x)
$$

where $F(x)=0$ defines a smooth surface in $\mathbb{P}^{3}$. The symmetrizer constructions and results in [14] illustrated above work equally well for a regular sequence. Thus, for the integral element defined by a surface variation, there is the additional constraint that the ideal generated by a regular sequence be a Jacobian ideal.

Although we shall not give the argument (using, as usual, Macaulay's theorem), it may be proved that an integral element $E$ defined by a regular sequence is maximal.

We set $\operatorname{dim} E=p$ and denote by

$$
G_{p}(\text { surfaces }) \subset G_{p}(R S) \subset G_{p}(I)
$$

the integral elements corresponding respectively to smooth surfaces, to regular sequences in $\mathbb{P}^{3}$, and general integral elements of the differential ideal generated by $I$. As just noted, the first inclusion is strict. The following would seem to be an interesting question:

$$
\text { Is an integral element } E \in G_{p}(R S) \text { ordinary (cf. (2.11) above)? }
$$

\subsection{The derived flag of the EDS associated to a VHS}

We shall prove the

(3.26) Theorem. Let $D$ be a period domain for polarized Hodge structures of weight $n \geqq 2$ and where all $h^{p, q} \neq 0$. Denote by $I \subset T^{*} D$ the Pfaffian system given by the infinitesimal period relations. Then for the derived system

$$
I_{[m]}=0 \quad \text { for } \quad m \geqq \frac{\log n}{\log 2} .
$$

Corollary. When $n \geqq 2$, I has no completely integrable subsystem. 
Remarks. ( $i$ ) This corollary roughly means that there are no finite equations satisfied by integral manifolds of $I$. More precisely, locally there are no non-constant functions $f$ on open subsets $\mathcal{U}$ of $D$ such that, for a point $F \in \mathcal{U}$, all local integral manifolds of $I$ passing through $F$ lie in the level set $f=$ const.

(ii) The theorem actually is valid on the dual classifying space $\check{D}$. Over $\check{D}$ there are Pfaffian systems $I(k)$ defined for $k \geqq 1$ by

$$
d F^{p} \subseteq F^{p-k} .
$$

For the VHS Pfaffian system, $I=I(1)$. We shall establish a stronger version of the theorem by showing that:

If all $h^{p, q} \neq 0$, then

$$
I_{[m]} \subseteq I\left(2^{m}\right) .
$$

Moreover, if $n \geqq 2$ and all $h^{p, q} \geqq 2$ then equality holds in (3.28).

(iii) We shall also see that the above result is sharp. The first case where some $h^{p, q}=0$ but $I \neq T^{*} D$ occurs when $n=4$ and $h^{4,0} \neq 0, h^{3,1} \neq 0$ but $h^{2,2}=0$. Then $I_{[\infty]} \neq 0$. The finite equations satisfied by a VHS may be described as follows: Denote by $\operatorname{Gr}\left(f^{2}, H_{\mathbb{C}}\right)$ the Grassmanian of $f^{2}$-dimensional planes in $H_{\mathbb{C}}$. Then, since $d F^{3} \equiv 0 \bmod F^{3}$, the connected integral manifolds of $I$ lie in the fibres of the obvious map

$$
\check{D} \rightarrow \operatorname{Gr}\left(f^{2}, H_{\mathbb{C}}\right) .
$$

(iv) The simplest interesting case where equality fails to hold in (3.28) is when $n=4$ and $h^{4,0}=1$. We shall see below that this is reflected in the structure of the derived flag. The conclusion will be: Among 4 -folds with $h^{4,0} \neq 0$, Calabi-Yau varieties are distinguished by the behaviour of the derived flag associated to the infinitesimal period relation.

Proof. In order not to have the notational complexity obscure the basic idea, we shall give the argument in the case $n=4$. From this we hope that the pattern for the general case will be evident. The assumption that all $h^{p, q} \neq 0$ is equivalent to the positive dimensionality of all graded quotients in the filtrations

$$
F^{4} \subset F^{3} \subset F^{2} \subset F^{1} \subset F^{0}=H_{\mathbb{C}}
$$

corresponding to a point of $\check{D}$. We consider adapted frame fields

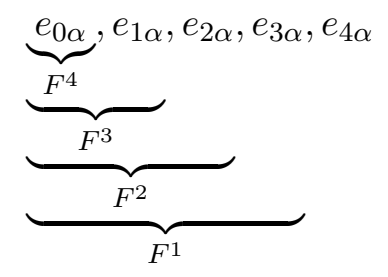

satisfying

$$
Q\left(e_{i \alpha}, e_{j \beta}\right)= \begin{cases}1 & \text { if } i+j=4, \alpha=\beta, \\ 0 & \text { otherwise }\end{cases}
$$

As usual we write, using summation convention,

$$
d e_{i \alpha}=\theta_{i \alpha}^{j \beta} e_{j \beta},
$$


where

$$
d \theta_{i \alpha}^{j \beta}=\theta_{i \alpha}^{k \gamma} \wedge \theta_{k \gamma}^{j \beta} .
$$

The forms $\theta_{i \alpha}^{j \beta}$ for $j \geqq i+1$ are semi-basic for the fibering $G_{\mathbb{C}} \rightarrow \check{D}$; it is only expressions in these that will have intrinsic meaning in the final formulas. We will use the notation $\equiv$ for congruence modulo the $\theta_{i \alpha}^{j \beta}$ for $j \leqq i$. The system $I$ is given by

$$
I=\left\{\theta_{i \alpha}^{j \beta}: j \geqq i+2\right\},
$$

where the brackets are used to denote the span.

The exterior derivative of (3.29) gives

$$
\theta_{i \alpha}^{4-j \beta}+\theta_{j \beta}^{4-i \alpha}=0 .
$$

For later reference we write this out in detail

$$
\left\{\begin{aligned}
\theta_{0 \alpha}^{4 \beta}+\theta_{0 \beta}^{4 \alpha}=0, & \theta_{1 \alpha}^{3 \beta}+\theta_{1 \beta}^{3 \alpha}=0, \\
\theta_{0 \alpha}^{3 \beta}+\theta_{1 \beta}^{4 \alpha}=0, & \theta_{1 \alpha}^{2 \beta}+\theta_{2 \beta}^{3 \alpha}=0, \\
\theta_{0 \alpha}^{2 \beta}+\theta_{2 \beta}^{4 \alpha}=0, & \\
\theta_{0 \alpha}^{1 \beta}+\theta_{3 \beta}^{4 \alpha}=0 . &
\end{aligned}\right.
$$

The 1-forms $\theta_{i \alpha}^{j \beta}$ are all non-zero and are linearly independent modulo these relations; the first statement uses the assumption that all $h^{p, q} \neq 0$. From (3.30), for $j \geqq i+1$

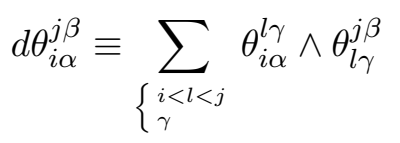

which by (3.31) gives the inclusion

$$
\left\{\theta_{i \alpha}^{j \beta}\right\}_{j \geqq i+3} \subseteq I_{[1]} .
$$

Indeed, if $j \geqq i+3$ then if $i<l<j$ we must have either $l-i \geqq 2, j-l \geqq 2$ or both. Now we use (3.31). Further, from (3.34)

$$
d \theta_{i \alpha}^{i+2 \beta} \equiv \sum_{\gamma} \theta_{i \alpha}^{i+1, \gamma} \wedge \theta_{i+1 \gamma}^{i+2 \beta} .
$$

Using (3.33) we may verify that no linear combination of the $\theta_{i \alpha}^{i+2 \beta}$ is in $I_{[1]}$. Thus we have equality in the above inclusion; i.e.

$$
\left\{\theta_{0 \alpha}^{3 \beta}, \theta_{0 \alpha}^{4 \beta}, \theta_{1 \alpha}^{4 \beta}\right\}=I_{[1]} .
$$

We next claim that

$$
I_{[2]}=0 .
$$

Indeed, denoting by $\equiv$ congruence modulo the algebraic ideal generated by $I_{[1]}$, by $(3.34)$ we have

$$
\left\{\begin{aligned}
d \theta_{0 \alpha}^{3 \beta} & \equiv \theta_{0 \alpha}^{1 \gamma} \wedge \theta_{1 \gamma}^{3 \beta}+\theta_{0 \alpha}^{2 \gamma} \wedge \theta_{2 \gamma}^{3 \beta} \\
d \theta_{0 \alpha}^{4 \beta} & \equiv \theta_{0 \alpha}^{2 \gamma} \wedge \theta_{2 \gamma}^{4 \beta} \\
d \theta_{1 \alpha}^{4 \beta} & \equiv \theta_{1 \alpha}^{3 \gamma} \wedge \theta_{3 \gamma}^{4 \beta}+\theta_{2 \alpha}^{2 \gamma} \wedge \theta_{2 \gamma}^{4 \beta}
\end{aligned}\right.
$$

no linear combination of the RHS is $\equiv 0$. 
Denoting by $\theta_{i}^{j}$ the matrix $\left\|\theta_{i \alpha}^{j \beta}\right\|$, the general pattern is

$$
\begin{array}{lll}
I=\left\{\theta_{i}^{j}: j \geqq i+1\right\}, & \text { i.e. } & j-i>1=2^{0}, \\
I_{[1]} \subseteq\left\{\theta_{i}^{j}: j \geqq i+3\right\}, & \text { i.e. } & j-i>2=2^{1}, \\
I_{[2]} \subseteq\left\{\theta_{i}^{j}: j \geqq i+5\right\}, & \text { i.e. } & j-i>4=2^{2}, \\
\vdots & & \\
I_{[k]} \subseteq\left\{\theta_{i}^{j}: j-i>2^{k}\right\} . &
\end{array}
$$

To show that equality holds, we must analyze in general the relations such as (3.33) that arise from $Q\left(F^{p}, F^{n-p+1}\right)=0$. The analysis is an extension of that given above when $n=4$, the one difference being the alteration of signs with the parity of $n$. We shall not write out the argument here. Rather we shall look more closely at the $n=4$ case when $h^{4,0}=1$ and when $h^{2,2}=0$.

Example: $h^{4,0}=1$. In this case $\theta_{0 \alpha}^{4 \beta}=0$ (only $\beta=\alpha$ occurs and we have $\theta_{0 \alpha}^{4 \alpha}=0$ by the first equation in (3.33)), and thus

$$
I \cap I(4)=0 .
$$

Conversely, if this relation holds then $h^{4,0}=1$.

Example: $h^{2,2}=0$. In this case

$$
\theta_{i \alpha}^{2 \beta}=0, \quad \theta_{2 \alpha}^{j \beta}=0, \quad i=0,1 \quad \text { and } \quad j=3,4 .
$$

Now

$$
d \theta_{1 \alpha}^{3 \beta} \equiv \theta_{1 \alpha}^{k \gamma} \wedge \theta_{k \gamma}^{3 \beta}
$$

and thus

$$
I_{[1]}=\left\{\theta_{0 \alpha}^{3 \beta}, \theta_{0 \alpha}^{4 \beta}, \theta_{1 \alpha}^{3 \beta}, \theta_{1 \alpha}^{4 \beta}\right\}
$$

We claim that

$$
I_{[2]}=I_{[1]} \cdot
$$

This will imply that $I_{[\infty]}=I_{[1]}$ and will verify the claim that leaves of the foliation given by $I_{[\infty]}$ are the fibres of $\check{D} \rightarrow \operatorname{Gr}\left(f^{2}, H_{\mathbb{C}}\right)$.

Continuing to denote by $\equiv$ equivalence modulo the algebraic ideal generated by $I_{[1]}$, from (3.34) we have

$$
\begin{aligned}
& d \theta_{0 \alpha}^{3 \beta} \equiv \theta_{0 \alpha}^{1 \gamma} \wedge \theta_{1 \gamma}^{3 \beta} \equiv 0, \\
& d \theta_{0 \alpha}^{4 \beta} \equiv \theta_{0 \alpha}^{1 \gamma} \wedge \theta_{1 \gamma}^{4 \beta}+\theta_{0 \alpha}^{3 \gamma} \wedge \theta_{3 \gamma}^{4 \beta} \equiv 0,
\end{aligned}
$$

and similarly for $d \theta_{1 \alpha}^{3 \beta}$ and $d \theta_{1 \alpha}^{4 \beta}$.

We are not aware of a geometric example where $h^{4,0} \neq 0, h^{3,1} \neq 0$ but $h^{2,2}=0$. There are of course geometric examples of necessarily rigid CY 3-folds where $h^{3,0}=1, h^{2,1}=0$. 


\section{Universal cohomology associated to a homogeneous Pfaffian system}

\subsection{EDS aspects of homogeneous Pfaffian systems}

In this section all Lie groups and Lie algebras will be real and connected. Let $G$ be a Lie group, $V \subset G$ a closed subgroup and

$$
M=G / V
$$

the resulting homogeneous space. We denote by $\mathcal{G}, \mathfrak{v}$ the Lie algebras respecively of $G, V$.

Definition. A homogeneous Pfaffian system is given by a $G$-invariant sub-bundle $I \subset T^{*} M$.

Equivalently, $I^{\perp}=: W \subset T M$ is a $G$-invariant distribution. Since $V$ is connected, this in turn is given by an ad-v invariant subspace

$$
\mathfrak{w} \subset \mathcal{G} / \mathfrak{v} \text {. }
$$

If $\tilde{\mathfrak{w}} \subset \mathcal{G}$ is the inverse image of $\mathfrak{w}$ under the projection of $\mathcal{G} \rightarrow \mathcal{G} / \mathfrak{v}$, ad- $\mathfrak{v}$ invariance is equivalent to $[\mathfrak{v}, \tilde{\mathfrak{w}}] \subseteq \tilde{\mathfrak{w}}$.

For any subspace $\mathfrak{a} \subset \mathfrak{w}$, the condition

$$
[\mathfrak{a}, \mathfrak{a}] \subseteq \mathfrak{v}
$$

is well-defined; the inverse image $\tilde{\mathfrak{a}} \subset \mathcal{G}$ of $\mathfrak{a}$ should satisfy $[\tilde{\mathfrak{a}}, \tilde{\mathfrak{a}}] \subseteq \mathfrak{v}$.

(4.2) Proposition. The G-invariant integral elements of a homogeneous Pfaffian system are given by the $G$-translates of subspaces $\mathfrak{a} \subset \mathfrak{w}$ that satisfy (4.1).

Proof. For the projection $G \stackrel{\pi}{\rightarrow} M$ we have that $\pi^{*} I$ is a $G$-invariant sub-bundle of $T^{*} G$; it is in fact given by the translates of

$$
\mathfrak{i}=\mathfrak{w}^{\perp} \subset \check{\mathcal{G}} .
$$

For $\theta \in \mathfrak{i}$, the Maurer-Cartan equation gives, for $\zeta, \eta \in \mathcal{G}$,

$$
\langle d \theta, \zeta \wedge \eta\rangle=-\frac{1}{2}\langle\theta,[\zeta, \eta]\rangle .
$$

For the $G$-invariant subspaces $E \subset T M$ given by the translates of $\mathfrak{a} \subset \mathfrak{w}$, the condition for such a subspace $E$ to be an integral element is therefore

$$
\left.d \theta\right|_{E}=0
$$

for all $\theta \in I$. By (4.3) this is equivalent to (4.1).

We now suppose that $M$ is a reductive homogeneous space so that there is an ad- $\mathfrak{v}$ invariant splitting

$$
\mathcal{G}=\mathfrak{p} \oplus \mathfrak{v} .
$$

Suppose that $\mathfrak{a} \subset \mathfrak{p}$ is a subspace that gives an abelian Lie-subalgebra; i.e.,

$$
[\mathfrak{a}, \mathfrak{a}]=0 .
$$

In particular, (4.1) is satisfied. Let $A=\exp \mathfrak{a}$ be the connected subgroup of $G$ corresponding to $\mathfrak{a}$. 
(4.5) Proposition (cf. Theorem 3.15 in [18]). The orbit $A \cdot(e V) \subset M$ is an integral manifold of $I$.

This is clear: The tangents to the $A$-orbit are the $A$ translates of $\mathfrak{a}$, and by (4.3) these are all integral elements.

The space $G_{p}(I)$ of $p$-dimensional integral elements is acted on by $G_{\mathbb{R}}$, and by the proposition every $E \in G_{p}(I)$ satisfying (4.4) is tangent to at least one integral manifold. This does not imply either that $(i) G_{p}(I)$ is smooth at $E$, or $(i i)$ that if $G_{p}(I)$ is smooth at $E$, then $E$ is an ordinary integral element; i.e., $I$ may not be involutive at $E$.

\subsection{Characteristic cohomology of the homogeneous Pfaffian system associated to a VHS}

Associated to a differential ideal $\mathcal{J}$ on a manifold $M$ are its characteristic cohomology groups $H_{\mathcal{J}}^{*}(M)$, defined to be the de Rham cohomology of the complex $\left(\Omega^{\bullet}(M) / \mathcal{J}, d\right)$ where $\Omega^{\bullet}(M)$ are the complex valued smooth forms on $M$. One may think of $H_{\mathfrak{J}}^{*}(M)$ as the universal cohomology groups induced on integral manifolds of $\mathcal{J}$. We refer to $[4,5]$ for a general reference as well as some examples of these groups.

In the case of a homogeneous Pfaffian system one may consider the complex $\left(\left(\Omega^{\bullet}(M) / \mathcal{J}\right)^{G}, d\right)$ of $G$-invariant forms. By standard arguments one has the

$$
\text { Proposition. } H^{*}\left(\left(\Omega^{\bullet}(M) / \mathcal{J}\right)^{G}, d\right) \cong H^{*}(\mathcal{G}, \mathfrak{v} ; \mathfrak{w}) \text {. }
$$

Here, $H^{*}(\mathcal{G}, \mathfrak{v} ; \mathfrak{w})$ is the Lie algebra cohomology of $(\mathcal{G}, \mathfrak{v})$ relative to $\mathfrak{w}$; everything is taken over the complex numbers. In the reductive case, which is the one we shall consider, this is defined as follows: First, we have

$$
\mathfrak{i}=\mathfrak{w}^{\perp} \subset \check{\mathfrak{p}} .
$$

To define the complex $C^{\bullet}(\mathcal{G}, \mathfrak{v} ; \mathfrak{w} ; \delta)$ we use the map

$$
\mathfrak{i} \rightarrow \mathfrak{i}_{2} \subset \wedge^{2} \check{\mathfrak{p}}
$$

defined by $\theta \rightarrow \theta_{2}$ where, for $\zeta, \eta \in \mathfrak{p}$

$$
\left\langle\theta_{2}, \zeta \wedge \eta\right\rangle=\langle\theta,[\zeta, \eta]\rangle \text {. }
$$

By the Maurer-Cartan equation, up to scaling these are just the 2 -forms $d \theta$ where $\theta \in I$, that together with $I$ generate the differential ideal J. Then we set

$$
C^{q}(\mathcal{G}, \mathfrak{v} ; \mathfrak{w} ; \delta)= \begin{cases}0, & \text { when } q=0, \\ (\check{\mathfrak{p}} / \mathfrak{i})^{\mathfrak{v}}, & \text { when } q=1, \\ \left(\wedge^{q} \check{\mathfrak{p}} / \wedge^{q-1} \check{\mathfrak{p}} \wedge \mathfrak{i}+\wedge^{q-2} \check{\mathfrak{p}} \wedge \mathfrak{i}_{2}\right)^{\mathfrak{v}}, & \text { for } q \geqq 2 .\end{cases}
$$

These are just the values at the identity coset $e V$ of the forms in $\left(\Omega^{q}(M) / \mathcal{J}^{q}\right)^{G}$. The map $\delta$ is just the exterior derivative, which when written out has the usual form of the differential in Lie algebra cohomology.

In applications, one frequently has a discrete subgroup $\Gamma$ acting properly discontinuously on $M$, and one is interested in the global invariants of maps

$$
f: S \rightarrow \Gamma \backslash M \text {. }
$$

There is then an induced map

$$
f^{*}: H^{*}(\mathcal{G}, \mathfrak{v} ; \mathfrak{w}) \rightarrow H^{*}(S) .
$$


We may think of the image of this map as giving the global topological invariants of maps $f: S \rightarrow \Gamma \backslash M$ that may be defined universally for all $\Gamma$ 's.

We now turn to the case of a period domain and use the complexified Lie algebras. $\mathcal{J}$ will be the differential ideal generated by $I \subset T^{1,0} D$ and $\bar{I} \subset T^{0,1} D$. In the classical case when $n=1$, $\mathfrak{w}=0$ and $V \subset G_{\mathbb{R}}$ is a maximal compact subgroup, and from what is known it may be inferred that

$H^{*}(\mathcal{G}, \mathfrak{v} ; \delta) \cong\left(\wedge^{*} \mathfrak{\mathfrak { p }}\right)^{\mathfrak{v}}$ is the space of invariants, and these are generated by the Chern forms of the Hodge bundles.

The argument will be given in several steps, some of which will carry over to the higher weight case.

(i) Since $D$ is a symmetric space, the symmetry about $e V$ is given by an element in $V$ which acts by -1 in the tangent space there. It follows that the invariant forms are all of even degree. In particular $\delta=0$, which gives the first statement in (4.9).

(ii) Next, using the unitary trick, and averaging, one infers that the space of invariant forms is isomorphic to the space of harmonic forms on the compact dual $\check{D}$, and this space is in turn isomorphic to $H^{*}(\check{D})$. This latter is known to be generated by the Chern classes of the universal vector bundle over $\check{D}$.

We will now show

(4.10) Theorem. For a period domain, the invariant forms that are orthogonal to the algebraic ideal generated by $I$ and $\bar{I}$ are all of type $(p, p)$.

Here, orthogonal means with respect to the canonical metrics constructed from the Hodge metrics in the Hodge bundles. Working in the orthogonal space is equivalent to working modulo the ideal in the space of all forms.

The first statement means that for a period domain the forms

$$
\left[\left(\wedge^{*-1} \check{\mathfrak{p}} \wedge \mathfrak{i}_{\mathbb{C}}\right)^{\perp}\right]^{\mathfrak{v}}
$$

are all of type $(p, p)$. This does not yet take into account the forms in $\wedge^{*-2} \check{\mathfrak{p}} \wedge \mathfrak{i}_{2}$.

Proof. At a reference point $F \in D$, we have a canonical representation

$$
\varphi: S^{1} \rightarrow \operatorname{Aut}\left(H_{\mathbb{R}}, Q\right)=G_{\mathbb{R}}
$$

obtained from a representation $\varphi_{\mathbb{C}}: \mathbb{C}^{*} \rightarrow \operatorname{Aut}\left(H_{\mathbb{C}}, Q\right)$ defined by

$$
\varphi_{\mathbb{C}}(z) v=z^{p} \bar{z}^{q} v, \quad v \in H^{p, q} .
$$

Restricted to $S^{1}=\{|z|=1\}, \varphi_{\mathbb{C}}(z)=\varphi(z)$ is real. We observe that $S^{1}$ is a subgroup of $V$; the Weil operator is just $C=\varphi(\sqrt{-1})$.

On the Lie algebra $\mathcal{G}$, we have the induced Hodge structure

$$
\mathcal{G}_{\mathbb{C}}=\oplus_{i} \mathcal{G}^{-i, i} .
$$

We then have

$$
\mathfrak{v}_{\mathbb{C}}=\mathcal{G}^{0,0}
$$

and

$$
\mathfrak{p}_{\mathbb{C}}=\mathfrak{p}^{+} \oplus \mathfrak{p}^{-}, \quad \mathfrak{p}^{-}=\overline{\mathfrak{p}}^{+},
$$


where

$$
\left\{\begin{array}{l}
\mathfrak{p}^{+}=\underset{i>0}{\oplus} \mathcal{G}^{-i, i} \cong T_{F}^{(1,0)} D \\
\mathfrak{p}^{-}=\underset{i<0}{\oplus} \mathcal{G}^{-i, i} \cong T_{F}^{(0,1)} D .
\end{array}\right.
$$

For the canonical Pfaffian system we have

$$
\mathfrak{i}_{\mathbb{C}}=\mathfrak{i}^{+} \oplus \mathfrak{i}^{-}, \quad \mathfrak{i}^{-}=\overline{\mathfrak{i}}^{+},
$$

where

$$
\left\{\begin{array}{l}
\mathfrak{i}^{+}=\check{\mathfrak{G}}^{-1,1} \subset \check{T}_{F}^{1,0} D, \\
\mathfrak{i}^{-}=\check{\mathfrak{G}}^{1,-1} \subset \check{T}_{F}^{0,1} D .
\end{array}\right.
$$

Thus the orthogonal complement to $\wedge^{*-1} \check{\mathfrak{p}}_{\mathbb{C}} \wedge \mathfrak{i}_{\mathbb{C}}$ are the forms of positive degree in

$$
\wedge^{*}\left(\check{\mathfrak{G}}^{-1,1} \oplus \check{\mathfrak{G}}^{1,-1}\right) \cong \wedge^{*} \check{\mathfrak{G}}^{-1,1} \otimes \wedge^{*} \check{\mathfrak{G}}^{1,-1} .
$$

On $\mathcal{G}^{-1,1}, \operatorname{Ad} \varphi(z)$ acts by $z^{-1} \bar{z}=z^{2} ;$ on $\mathcal{G}^{1,-1}$ it acts by $z \bar{z}^{-1}=z^{-2}$. Thus on $\wedge^{p} \check{\mathcal{G}}^{-1,1} \otimes \wedge^{q} \check{\mathcal{G}}^{1,-1}$, $\operatorname{Ad} \varphi(z)$ acts by $z^{2(p-q)}$. Since $\varphi(z) \in V$ for $z \in S^{1}$, we see that any ad $\mathfrak{v}$-invariant forms must be of type $(p, p)$.

As mentioned in the introduction, we expect that (4.10) may be the first step in showing that the $G_{\mathbb{R}}$-invariant forms modulo the differential ideal generated by $I$ are generated by the Chern forms of the Hodge bundles ${ }^{7}$. Since, except in the classical case when $I=0$, the invariant forms modulo the algebraic ideal generated by $I$ definitely contain more than the Chern forms, the integrability conditions will have to enter in an essential way. In fact, they enter in the proof of the following

(4.11) Theorem. The Chern forms of the Hodge filtration bundles satisfy

$$
c_{i}\left(F^{p}\right) c_{j}\left(F^{n-p}\right)=0 \quad \text { if } \quad i+j>h^{p, n-p} .
$$

Proof. The argument will proceed in several steps, the key one where integrability is used being (4.17) below.

Step one. For a $d \times d$ matrix $A$ we set

$$
\chi_{A}(t)=: \operatorname{det}(A-t I)=t^{d}+c_{1}(A) t^{d-1}+\cdots+c_{d}(A) .
$$

Then we have

$$
\text { Lemma. If } A, B \text { are } d \times d \text { matrices with } A B=0 \text {, then }
$$

$$
c_{i}(A) c_{j}(B)=0 \quad \text { if } i+j>d .
$$

Proof. We have

$$
\chi_{A}(t) \chi_{B}(u)=\operatorname{det}(A-t I) \operatorname{det}(B-u I)=\operatorname{det}(-t B-u A+u t I),
$$

and all terms in $\operatorname{det}(-t B-u A+u t I)$ have degree at least $d$ in $u, t$. From

$$
\chi_{A}(t) \chi_{B}(u)=\sum_{i, j} c_{i}(A) c_{j}(B) t^{d-i} u^{d-j}
$$

we have $c_{i}(A) c_{j}(B)=0$ if $(d-i)+(d-j)<d$; i.e. if $i+j>d$.

\footnotetext{
${ }^{7}$ As mentioned in the introduction, this result has now been proved. The integrability conditions play the central role in the argument, which is representation-theoretic in nature.
} 
Step two. We let $E \subset \mathcal{G}^{-1,1}$ be an integral element at a point $F \in D$. Then we have $\check{E}$-valued maps

$$
F^{p} / F^{p+1} \stackrel{A_{p}}{\longrightarrow} F^{p-1} / F^{p}
$$

where we think of $A_{p}$ as a matrix of size $h^{p, n-p} \times h^{p-1, n-p+1}$ whose entries are $\check{E}$. The integrability conditions are

$$
A_{p-1} \cdot A_{p}=0
$$

where we are multiplying matrices using the wedge product of their entries.

Using the dualities induced by $Q$ and denoting the transpose of a matrix $A$ by $A^{*}$ we have a commutative diagram

$$
\begin{array}{ccc}
\stackrel{\left.F^{p-1} / F^{p}\right)}{\stackrel{A_{p}^{*}}{\longrightarrow}} & \left(F^{p} / F^{p+1}\right) \\
2 \| & & 2 \| \\
F^{n-p+1} / F^{n-p+2} & \stackrel{A_{n-p+1}}{\longrightarrow} & F^{n-p} / F^{n-p+1} .
\end{array}
$$

On $F^{p}$ we have an Hermitian metric induced by $(v, w)=Q(C v, \bar{w})$.

Lemma. Up to a constant, the curvature matrix of the metric connection is given by

$$
\Theta_{F^{p}}=\left(\begin{array}{cc}
\bar{A}_{p}^{*} A_{p} & 0 \\
0 & 0
\end{array}\right) .
$$

The notation means that we write the orthogonal direct sum decomposition

$$
F^{p}=H^{p, n-p} \oplus F^{p+1} .
$$

A consequence of $(4.14)$ is

$$
c_{k}\left(F^{p}\right)=0 \quad \text { for } \quad k>h^{p, n-p} .
$$

Proof of the lemma. $F^{p}$ is a sub-bundle of the flat bundle $F^{0}=H_{\mathbb{C}}$ with the induced metric. In this situation it is known [16] that the curvature of the metric connection in $F^{p}$ is up to a constant given by

$$
\bar{\Pi}_{F^{p} / H}^{*} \cdot \Pi_{F^{p} / H},
$$

where $\Pi_{F^{p} / H}$ is the $2^{\text {nd }}$ fundamental form of $F^{p}$ in $H_{\mathbb{C}}$. In the case at hand the $2^{\text {nd }}$ fundamental form may be identified with $A_{p}$.

Remark. There is one subtlety here. Because of the sign alternation in the Hodge metrics

$$
(u, v)=(\sqrt{-1})^{p-q} Q(u, \bar{v}) \quad u, v \in H^{p, q}
$$

the usual principle that "curvatures decrease on holomorphic sub-bundles" does not hold for the Hodge bundles. For example, $c_{1}\left(F^{n}\right)>0$ on $\Theta_{F^{p}}$. However, the signs are not an issue for us here.

Step three. From (4.13) we have

$$
\bar{A}_{p}^{*} A_{p} A_{p+1} \bar{A}_{p+1}^{*}=0,
$$


where the multiplication of matrix entries is wedge product. For notational simplicity we omit the blocks of zeroes in the $\Theta_{F^{p}}$ 's, so that

$$
\begin{aligned}
& \Theta_{F^{p}}=\bar{A}_{p}^{*} A_{p}, \\
& \Theta_{F^{n-p}}^{*}=-A_{p+1} \bar{A}_{p+1}^{*} .
\end{aligned}
$$

Using (4.16) this gives the remarkable consequence

$$
\Theta_{F^{p}} \Theta_{F^{n-p}}^{*}=0
$$

of integrability. Since $\chi_{\Theta_{F}^{*}{ }^{n-p}}(t)=\chi_{\Theta_{F^{n-p}}}(t)$, by step one we see that $c_{i}\left(F^{p}\right) c_{j}\left(F^{n-p}\right)=0$ if $i+j>h^{p, n-p}$.

\section{5 "Expected" dimension counts for integral manifolds of an EDS}

An important aspect in algebraic geometry is that of "expected" dimension counts. Informally and in first approximation, this means counting the number of parameters of solutions to a system of algebraic equations, where "expected" means assuming some sort of "general position". When the solution varieties are also subject to differential constraints, the problem changes character in an interesting way. In this section we will discuss this for the EDS arising from VHS's.

To frame the general issue we assume given a diagram of regular mappings of complex manifolds

$$
\begin{array}{lll}
X & \stackrel{f}{\rightarrow} & A \\
\cup & & \cup \\
Y & \rightarrow & B
\end{array}
$$

where $f$ is an immersion and $Y=f^{-1}(B)$. Then

$$
\text { "expected" } \operatorname{codim}_{X} Y=\operatorname{codim}_{A} B=\operatorname{rank}(T A / T B),
$$

where it is understood that $T A / T B$ is restricted to $B$. If $Y$ is non-empty, then the actual codimension satisfies

$$
\operatorname{codim}_{X} Y \leqq \operatorname{rank}(T A / T B)
$$

with equality holding when $f(X)$ meets $B$ transversely.

Now suppose that $I \subset T^{*} A$ is a holomorphic Pfaffian system and $f: X \rightarrow A$ is an integral manifold of $I$. Let $W=I^{\perp}$ be the corresponding distribution. Then for the normal bundles we have that

$$
f_{*}: T X / T Y \rightarrow W / W \cap T B
$$

is injective, so that the above may be refined to

$$
\operatorname{codim}_{X} Y \leqq \operatorname{rank}(W / W \cap T B) \leqq \operatorname{rank}(T A / T B) .
$$

Informally we may say that: Subjecting $f: X \rightarrow A$ to a differential constraint decreases the codimension of $Y=f^{-1}(A)$ in $X$. By "decreases" we mean that $\operatorname{codim}_{X} Y$ is less than the "expected" codimension in the absence of differential constraints, as explained above. 
However, when we take into account integrability a still further refinement of (5.3) may be expected. This is because in general integral elements of $I$ may be expected to have dimension smaller, frequently much less, than rank $W$.

Rather than discuss the general aspects of this, we turn to a special case that is geometrically motivated. Let $D$ be a period domain for polarized Hodge structures of even weight $n=2 m$. At a reference Hodge structure $F \in D$ we let $\zeta \in H_{\mathbb{R}} \cap H^{m, m}$ be a real vector of type $(m, m)$.

Definition. The Noether-Lefschetz locus is the subvariety $D_{\zeta} \subset D$ where $\zeta$ remains of type $(m, m)$.

(5.4) Proposition. Let $G_{\zeta} \subset G_{\mathbb{R}}$ be the subgroup fixing $\zeta$ up to scaling. Then $D_{\zeta}=G_{\zeta} \cdot F$ is the $G_{\zeta}$-orbit of $F$. It is a homogeneous complex sub-manifold of $D$ of codimension given by

$$
\operatorname{codim}_{D} D_{\zeta}=h^{(2 m, 0)}+\cdots+h^{(m+1, m-1)} .
$$

Proof. This is a matter of routine checking. Setting $h_{k}=h^{(2 m-k, k)}$, we have first

$$
\left\{\begin{array}{l}
G_{\mathbb{R}} \cong O(a, b), \quad a+b=h_{0}+\cdots+h_{2 m}, \\
V \cong \mathcal{U}\left(h_{0}\right) \times \cdots \times \mathcal{U}\left(h_{m-1}\right) \times O\left(h_{m}\right)
\end{array}\right.
$$

Next, depending on whether $m$ is even or odd, we have $G_{\zeta} \cong O(a-1, b)$ or $O(a, b-1)$. Finally, the same linear algebra argument that shows that $G_{\mathbb{R}}$ acts transitively on $D$ shows that $G_{\zeta}$ acts transitively on $D_{\zeta}$ and

$$
D_{\zeta}=G_{\zeta} / V_{\zeta}
$$

where

$$
V_{\zeta}=G_{\zeta} \cap V \cong \mathcal{U}\left(h_{0}\right) \times \cdots \times \mathcal{U}\left(h_{m-1}\right) \times O\left(h_{m}-1\right) .
$$

From this we may conclude the above codimension count.

For fixed $\zeta \in H_{\mathbb{R}} \cap H^{(m, m)}$ and at a variable point in $D$ we write the Hodge decomposition of $\zeta$ as

$$
\left\{\begin{array}{l}
\zeta=\zeta^{2 m, 0}+\cdots+\zeta^{m, m}+\cdots+\zeta^{0,2 m}, \\
\zeta^{\overline{2 m-p, p}}=\zeta^{p, 2 m-p} .
\end{array}\right.
$$

Then $D_{\zeta}$ is defined by the equations

$$
\zeta^{m-1, m+1}=\cdots=\zeta^{0,2 m}=0 .
$$

The above proposition says that these equations are independent and define $D_{\zeta}$ as a smooth complex submanifold of $D$.

At a point $F \in D$ we let

$$
\left\{\begin{array}{l}
E \subset T_{F} D \quad \text { be an integral element of } I, \\
E_{\zeta}=E \cap T_{F} D_{\zeta} .
\end{array}\right.
$$

For $\varphi \in \mathcal{G}^{-1,1} \subset \oplus \operatorname{Hom}\left(H^{2 m-p, p}, H^{2 m-p-1, p+1}\right)$ we write $\varphi=\varphi_{0}+\cdots+\varphi_{2 m-1}$ where $\varphi_{p} \in$ $\operatorname{Hom}\left(H^{2 m-p, p}, H^{2 m-p-1, p+1}\right)$ and $\varphi_{p}$ and $\varphi_{2 m-p-1}$ are dual. Then

$$
E_{\zeta}=\left\{\varphi \in E: \varphi_{m}(\zeta)=0 \text { in } H^{m-1, m+1}\right\} .
$$


This is equivalent to

$$
E_{\zeta}=\left\{\varphi \in E: Q(\eta, \varphi(\zeta))=0 \text { for all } \eta \in H^{m+1, m-1}\right\} .
$$

Thus, without taking the integrability conditions into account we have

$$
\operatorname{codim}_{E} E_{\zeta} \leqq h^{m+1, m-1} .
$$

However, due to the integrability conditions the equations (5.5) may not be independent. In order to illustrate the essential point, we begin by considering the first non-trivial case $m=2$. For any $\varphi \in E_{\zeta}, \psi \in E$ and $\omega \in H^{4,0}$, using that $E$ is an integral element so that $\varphi$ and $\psi$ commute,

$$
Q(\varphi(\omega), \psi(\zeta))=-Q(\psi \varphi(\omega), \zeta)=-Q(\varphi \psi(\omega), \zeta)=Q(\psi(\omega), \varphi(\zeta))=0 ;
$$

i.e., for each $\psi \in E$ the linear equations

$$
Q(\eta, \psi(\zeta))=0, \quad \eta \in H^{3,1}
$$

that define the condition that $\psi \in E_{\zeta}$ are decreased in rank by

$$
\sigma_{\zeta}=: \operatorname{dim} \operatorname{Im}\left\{E_{\zeta} \otimes H^{4,0} \rightarrow H^{3,1}\right\} .
$$

We thus have the

Proposition. For $\sigma_{\zeta}$ defined as above $\operatorname{codim}_{E} E_{\zeta} \leqq h^{1,3}-\sigma_{\zeta}$.

The general case goes as follows: With $E_{\zeta}$ defined as above, for each $p$ with $0 \leqq p \leqq m-2$ we consider the maps

$$
\kappa_{\zeta}^{p}: E \otimes \operatorname{Sym}^{p-m+1} E \otimes H^{2 m-p, p} \rightarrow H^{m+1, m-1}
$$

and we set

$$
\sigma_{\zeta}=\operatorname{dim}\left\{\operatorname{span}\left(\operatorname{Images} \kappa_{\zeta}^{p}\right) \text { for } 0 \leqq p \leqq m-2\right\} .
$$

Then the straightforward extension of the above argument gives the

$$
\begin{aligned}
& \text { (5.9) Proposition. For } \sigma_{\zeta} \text { defined by (5.8), we have } \\
& \quad \operatorname{codim}_{E} E_{\zeta} \leqq h^{m-1, m+1}-\sigma_{\zeta} \text {. } \\
& \text { In algebro-geometric terms, this says that the "richer" the multiplicative structure in the } 1^{\text {st }} \\
& \text { order variation of the Hodge structure, the smaller the codimension of Noether-Lefschetz loci. }
\end{aligned}
$$

Remark. The above is predicated on the implicit assumption that, for a variation of Hodge structure $S \rightarrow \Gamma \backslash D$, we have that the Noether-Lefschetz locus $S_{\zeta} \subset S$ is reduced, so that for general points of a component of $S_{\zeta}$ we have $\operatorname{codim}_{S}\left(S_{\zeta}\right)=\operatorname{codim}_{T_{s} S}\left(T_{s} S_{\zeta}\right)$. For far as we know, there are not yet any examples coming from algebraic geometry where this assumption is not satisfied, although we feel that such examples may be expected.

Example. We consider a smooth hypersurface

$$
X \subset \mathbb{P}^{5}
$$

of degree $d \geqq 6$ and which contains a 2 -plane $P \cong \mathbb{P}^{2}$.

(5.10) Theorem. For the primitive part of the fundamental class $[P] \in H^{4}(X, \mathbb{Z})$ of $P$, we have equality in (5.9). 
Proof. We will denote by $V \cong \mathbb{C}^{6}$ a vector space with coordinates $x_{1}, \ldots, x_{6}$ such that $X \subset \mathbb{P} \check{V}$ is given by an equation

$$
F(x)=0
$$

where $F \in V^{d}=: \operatorname{Sym}^{d} V$ is a homogeneous polynomial of degree $d \geqq 6$. It is well-known, and will be proved below, that

(5.11) At a general $X$ containing a 2-plane $P$, the locus of all degree $d$ hypersurfaces $X^{\prime}$ close to $X$ and containing a 2-plane $P^{\prime}$ close to $P$ is smooth and of codimension $\frac{(d+1)(d+2)}{2}-$ $\operatorname{dim} \operatorname{Gr}(3,6)$ in the space of all degree d hypersurfaces in $\mathbb{P} \check{V}$.

Let $P$ be given by $x_{1}=x_{2}=x_{3}=0$ so that

$$
F(x)=\sum_{i=1}^{3} x_{i} G_{i}(x),
$$

where $G_{i} \in V^{d-1}$. Denote by

$$
F_{t}(x)=F(x)+t \dot{F}(x), \quad \dot{F} \in V^{d},
$$

a $1^{\text {st }}$ order perturbation of $F$. The condition that $P$ move to $1^{\text {st }}$ order to a 2 -plane $P_{t} \subset X_{t}=$ $\left\{F_{t}(x)=0\right\}$ is that

$$
\dot{F}=\sum_{i} x_{i} \dot{G}_{i}+\sum_{i} l_{i} G_{i}
$$

where $\dot{G}_{i} \in V^{d-1}$ is the tangent to a $1^{\text {st }}$ order variation of $G_{i}$ and the $l_{i} \in V$ are linear forms. We will use the notation $\left(X^{\prime}, P^{\prime}\right)$ for the $1^{\text {st }}$ order perturbation of $(X, P)$.

We will denote by $\left(H_{1}, \ldots, H_{m}\right)$ the ideal generated by forms $H_{1}, \ldots, H_{m}$, and by $\left(H_{1}, \ldots, H_{m}\right)_{k}$ the degree $k$ part of that ideal. Thus, the subspace of $V^{d}$ that gives the $1^{\text {st }}$ order deformations $\left(X^{\prime}, P^{\prime}\right)$ of $(X, P)$ is

$$
\left(x_{1}, x_{2}, x_{3}, G_{1}, G_{2}, G_{3}\right)_{d} .
$$

The Jacobian ideal is

$$
J=\left(\partial_{x_{1}} F, \ldots, \partial_{x_{6}} F\right) .
$$

For references to Jacobian ideals and the polynomial description of the cohomology of hypersurfaces we suggest $[1,7]$, and [13]. The tangent space to $1^{\text {st }}$ order deformations of the projective equivalence class of $X$ is given by

$$
T=V^{d} / J_{d}
$$

With this identification, by what was said above the subspace $T_{P} \subset T$ giving $1^{\text {st }}$ order deformations of equivalence classes of pairs $(X, P)$ is given by

$$
T_{P}=\left(x_{1}, x_{2}, x_{3}, G_{1}, G_{2}, G_{3}\right)_{d} / J_{d} .
$$

A basic identification is

$$
H^{4-p, p}(X)_{\text {prim }}=V^{(p+1) d-6} / J_{(p+1) d-6} .
$$

Thus, for example

$$
H^{4,0}(X) \cong V^{d-6} .
$$


Using the identification $T=V^{d} / J_{d}$, the differential of the period mapping

$$
T \rightarrow \underset{p}{\oplus} \operatorname{Hom}\left(H^{4-p, p}(X), H^{4-p-1, p+1}(X)\right)
$$

is given by multiplication in the ring $V \bullet / J_{\bullet}$; i.e. by

$$
V^{d} / J_{d} \otimes V^{(p+1) d-6} / J_{(p+1) d-6} \rightarrow V^{(p+2) d-6} / J_{(p+2) d-6} .
$$

We denote by $\zeta \in H^{2,2}(X)_{\text {prim }}$ the primitive part of the fundamental class $[P]$ of $P$.

Proposition. If we define

$$
T_{\zeta}=\left\{H \in V^{d} / J_{d}: H \cdot \zeta=0 \text { in } V^{4 d-6} / J_{4 d-6}\right\}
$$

then

$$
T_{\zeta}=T_{P}
$$

The inclusion $T_{P} \subseteq T_{\zeta}$ is clear geometrically: It means that if $(X, P)$ deforms to $1^{\text {st }}$ order to $\left(X^{\prime}, P^{\prime}\right)$ as above, then $\zeta$ deforms to a Hodge class $\zeta^{\prime} \in H^{2}\left(X^{\prime}\right)_{\text {prim. }}$. In fact, $\zeta^{\prime}=\left[P^{\prime}\right]_{\text {prim }}$. The proof of the reverse inclusion will come out indirectly from the argument to be given below. The assertion (5.11) is a consequence of (5.15).

We set

$$
V_{P}=\left.V\right|_{P}=V /\left(x_{1}, x_{2}, x_{3}\right)
$$

The proof of the theorem and the proposition will be based on known commutative algebra properties of the rings $V^{\bullet} / J_{\bullet}$ and $V_{P}^{\bullet} / J_{P, \bullet}$; namely there are perfect pairings

$$
\left\{\begin{array}{c}
(i) \quad V^{k} / J_{k} \otimes V^{6 d-12-k} / J_{6 d-12-k} \rightarrow V^{6 d-12} / J_{6 d-12} \cong \mathbb{C}, \\
(i i) \quad V_{P}^{k} /\left(G_{1}, G_{2}, G_{3}\right)_{P, k} \otimes V_{P}^{3 d-6-k} /\left(G_{1}, G_{2}, G_{3}\right)_{P, 3 d-6-k} \\
\rightarrow V_{P}^{3 d-6} /\left(G_{1}, G_{2}, G_{3}\right)_{P, 3 d-6} \cong \mathbb{C} .
\end{array}\right.
$$

Here, we denote by $\left(G_{1}, G_{2}, G_{3}\right)_{P, k}$ the degree $k$ part of the ideal generated by the $G_{i} \mid P$. The reason for $(5.16)$ is that $\left(x_{1}, x_{2}, x_{3}, G_{1}, G_{2}, G_{3}\right)$ is a regular sequence on $\mathbb{P}^{5}$, and this then implies that $\left.G_{1}\right|_{P},\left.G_{2}\right|_{P},\left.G_{3}\right|_{P}$ is a regular sequence on $P$. Then in general if $f_{1}, \ldots, f_{n}$ is a regular sequence on $\mathbb{P}^{n}$ where $\operatorname{deg} f_{i}=d_{i}$, there is a perfect pairing $V^{a} /\left(f_{1}, \ldots, f_{n}\right)_{a} \otimes V^{b} /\left(f_{1}, \ldots, f_{n}\right)_{b} \rightarrow$ $V^{a+b} /\left(f_{1}, \ldots, f_{n}\right)$ where $a+b=\sum_{i} d_{i}-n$.

The argument will proceed in five steps, the first of which is (5.13) above.

Step two. We have

$$
T_{P} H^{4,0}=\left(x_{1}, x_{2}, x_{3}, G_{1}, G_{2}, G_{3}\right)_{2 d-6} / J_{2 d-6} \subseteq V^{2 d-6} / J_{2 d-6} .
$$

This follows from (5.13) and (5.14) in the case $p=0$.

Step three. Denoting by $[P]$ the fundamental class of $P$, the map

$$
H^{2,2}(X) \stackrel{[P]}{\rightarrow} H^{4,4}(X) \cong \mathbb{C}
$$

may, using (ii) in (5.14) which gives an isomorphism $V^{3 d-6} /\left(G_{1}, G_{2}, G_{3}\right)_{P, 3 d-6} \cong \mathbb{C}$, be identified with

$$
V^{3 d-6} / J_{3 d-6} \rightarrow V_{P}^{3 d-6} /\left(G_{1}, G_{2}, G_{3}\right)_{P, 3 d-6} \cong \mathbb{C}
$$


Proof. Since $X$ is non-singular the restrictions $\left.G_{i}\right|_{P}$ have no common zeroes and hence form a regular sequence. The corresponding Koszul resolution of $\mathcal{O}_{P}$ then gives

$$
0 \rightarrow \mathcal{O}_{P}(-3(d-1)) \rightarrow \underset{3}{\oplus} \mathcal{O}_{P}(-2(d-1)) \rightarrow \underset{3}{\oplus} \mathcal{O}_{P}(-(d-1)) \rightarrow \mathcal{O}_{P} \rightarrow 0 .
$$

Tensoring with $\mathcal{O}_{P}(3 d-6)$ gives, using $\Omega_{P}^{2} \cong \mathcal{O}_{P}(-3)$

$$
0 \rightarrow \Omega_{P}^{2} \rightarrow \underset{3}{\oplus} \mathcal{O}_{P}(d-4) \rightarrow \underset{3}{\oplus} \mathcal{O}_{P}(2 d-5) \rightarrow \mathcal{O}_{P}(3 d-6) \rightarrow 0,
$$

from which we infer that

$$
V_{P}^{3 d-6} /\left(G_{1}, G_{2}, G_{3}\right)_{P, 3 d-6} \cong H^{2}\left(P, \Omega_{P}^{2}\right) \cong \mathbb{C} .
$$

Moreover, under this isomorphism a generator of $V_{P}^{3 d-6} /\left(G_{1}, G_{2}, G_{3}\right)_{P, 3 d-6}$ maps to the fundamental class. Since the map $H^{2,2}(X) \stackrel{[P]}{\rightarrow} H^{4,4}(X)$ is given by evaluating a class in $H^{2,2}(X)$ on the fundamental class of $P$, by standard arguments we may infer the assertion in Step 3.

Step four. We first observe that the map

$$
\zeta \otimes V^{d} / J_{d} \otimes T_{P} \otimes V^{d-6} \rightarrow V^{4 d-6} / J_{4 d-6} \cong \mathbb{C}
$$

is zero. Here, we recall that $\zeta \in V^{3 d-6} / J_{3 d-6}$ is the primitive part of $[P] \in H^{2,2}(X)$. We are using (5.14) that the action on cohomology of tangent vectors to deformations of equivalence classes of hypersurfaces in $\mathbb{P}^{5}$ is given by multiplication in the ring $V^{\bullet} / J_{\bullet}$. The fact that the above map is zero results from the definition of $T_{\zeta}$ as the kernel of the map

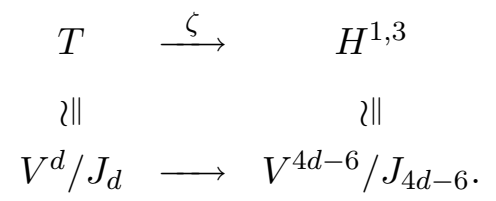

Then the claim is that the map (5.17) may be identified with

$$
\left.T_{P} \cdot V^{d-6}\right|_{P} \in V_{P}^{3 d-6} /\left(G_{1}, G_{2}, G_{3}\right)_{P, 3 d-6} \cong \mathbb{C} .
$$

This follows from Step three above.

Step five. We now put everything together. To prove the theorem it will suffice to show that $T_{P}$ is the kernel of

$$
V^{d} / J_{d} \stackrel{\zeta}{\rightarrow} V^{4 d-6} / J_{4 d-6}
$$

This is because for all $S \in V^{d} / J_{d}$,

$$
R \cdot \zeta=0 \text { in } V^{6 d-6} / J_{6 d-6} \text { for all } R \in V^{2 d-6}
$$

is, by $(5.16)(i)$, equivalent to

$$
S \cdot \zeta=0 \text { in } V^{4 d-6} / J_{4 d-6} .
$$

By step four, (5.19) is in turn equivalent to

$$
\left.R S\right|_{P} \in\left(G_{1}, G_{2}, G_{3}\right)_{P, 3 d-6},
$$

which by (5.16) $(i i)$ is the same as the condition

$$
\left.S\right|_{P} \in\left(G_{1}, G_{2}, G_{3}\right)_{P, d} .
$$

This last statement is easily seen to be equivalent to

$$
S \in T_{P} .
$$




\section{Reprise}

We consider the case that arises in the case of a family of Calabi-Yau fourfolds. Thus, we assume that $h^{4,0}=1$ and denote by $\omega \in H^{4,0}$ a generator giving an isomorphism $H^{4,0} \cong \mathbb{C}$. Moreover, let $T \subset \mathcal{G}^{-1,1}$ be an integral element and, as would be the case for Calabi-Yau's, we assume that the map given by $(5.13)$

$$
T \rightarrow \operatorname{Hom}\left(H^{4,0}, H^{3,1}\right) \cong H^{3,1}
$$

is an isomorphism. Using this map we may identify $T$ with $H^{3,1}$ and denote its dimension by $m$ (for moduli).

Each $\zeta \in H^{2,2}$ defines a quadric

$$
Q_{\zeta} \in \operatorname{Sym}^{2} \check{T}
$$

given for $\theta, \theta^{\prime} \in T$ by

$$
\left\langle Q_{\zeta}, \theta \cdot \theta^{\prime}\right\rangle=Q\left(\theta \cdot \theta^{\prime} \omega, \zeta\right)
$$

The fact that this is symmetric in $\theta$ and $\theta^{\prime}$ is because $T$ is an integral element.

Denote by $T_{\zeta} \subset T$ the intersection of $T$ with the tangent space to the Noether-Lefschetz locus $D_{\zeta} \subset D$.

(5.20) Proposition. We have

$\operatorname{codim} T_{\zeta}=\operatorname{rank} Q_{\zeta}$

Moreover, in the case of the family of hypersurfaces $X \subset \mathbb{P}^{5}$ of degree six and where $\zeta$ is the primitive part of the fundamental class of a plane $P \subset X$, we have $T_{P}=T_{\zeta}$.

Proof. We view $Q_{\zeta}$ as a map

$$
Q_{\zeta}: T \rightarrow \check{T}
$$

Then the proof of Proposition 5.7 gives

$$
T_{\zeta}=\left(\operatorname{Im} Q_{\zeta}\right)^{\perp}
$$

thus

$$
\operatorname{codim} T_{\zeta}=\operatorname{rank} Q_{\zeta}
$$

as desired.

For the case of $P \subset X \subset \mathbb{P}^{5}, \operatorname{deg} X=6$, we have

$$
T=V^{6} / J_{6}
$$

Moreover, we have seen in step three in the proof of (5.15) that

$$
T_{\zeta}=\operatorname{ker}\left\{V^{6} / J_{6} \rightarrow V_{P}^{6} /\left(G_{1}, G_{2}, G_{3}\right)_{P, 6}\right\} .
$$

Thus

$$
\operatorname{rank} Q_{\zeta}=\operatorname{dim} V_{P}^{6} /\left(G_{1}, G_{2}, G_{3}\right)_{P, 6}
$$


Now, from the Koszul calculation above

$$
0 \rightarrow \underset{3}{\oplus} V_{P} \stackrel{\left(G_{1}, G_{2}, G_{3}\right)}{\longrightarrow} V_{P}^{6}
$$

is exact. Thus

$$
\operatorname{rank} Q_{\zeta}=\operatorname{dim} V_{P}^{6}-3 \operatorname{dim} V_{P}=\left(\begin{array}{l}
8 \\
2
\end{array}\right)-3 \cdot 6=19 .
$$

On the other hand, the number of conditions for $X$ to contain a 2-plane is

$$
\operatorname{dim} V_{P}^{6}-\operatorname{dim} \operatorname{Gr}(3,6)=\left(\begin{array}{l}
8 \\
2
\end{array}\right)-9=19 .
$$

Remark. Let $X$ be a Calabi-Yau fourfold and $\zeta \in \operatorname{Hg}^{2}(X)_{\text {prim }}$ a Hodge class. We then have

(5.21) If the Hodge conjecture is true and $\operatorname{rank} Q_{\zeta}=h^{3,1}$ is maximal, then $X$ is defined over a number field.

This is because if $Q_{\zeta}$ is non-singular, then the Noether-Lefschetz locus for $\zeta$ will be 0dimensional. If $\zeta=[Z]$ is the class of an algebraic cycle, then $X$ is defined over a field $k$ of transcendence degree $\geqq 1$, and by standard arguments we may, after passing to a finite field extension, assume that $Z$ is also defined over $k$. The spread of $(X, Z)$ will then give a positive dimensional component to the Noether-Lefschetz locus of $\zeta$.

To disprove the consequence of the Hodge conjecture that Hodge classes are absolute, it would be sufficient to find a Calabi-Yau fourfold not defined over a number field and a Hodge class $\zeta$ such that $Q_{\zeta}$ is non-singular.

\section{References}

[1] Allaud E., Nongenericity of variations of Hodge structure for hypersurfaces of high degree, Duke Math. J. 129 (2005), 201-217, math.AG/0503346.

[2] Bryant R.L., Chern S.S., Gardner R.B., Goldschmidt H.L., Griffiths P.A., Exterior differential systems, Mathematical Sciences Research Institute Publications, Vol. 18, Springer-Verlag, New York, 1991.

[3] Bryant R.L., Griffiths P.A., Some observations on the infinitesimal period relations for regular threefolds with trivial canonical bundle, in Arithmetic and Geometry, Vol. II, Progr. Math., Vol. 36, Birkhäuser Boston, Boston, MA, 1983, 77-102.

[4] Bryant R.L, Griffiths P.A., Characteristic cohomology of differential systems. I. General theory, J. Amer. Math. Soc. 8 (1995), 507-596.

[5] Bryant R.L, Griffiths P.A., Characteristic cohomology of differential systems. II. Conservation law for a class of parabolic equations, Duke Math. J. 78 (1995), 531-676.

[6] Carlson J.A., Bounds on the dimension of variations of Hodge structure, Trans. Amer. Math. Soc. 294 (1986), 45-64, Erratum, Trans. Amer. Math. Soc. 299 (1987), 429.

[7] Carlson J.A., Donagi R., Hypersurface variations are maximal. I, Invent. Math. 89 (1987), 371-374.

[8] Carlson J.A., Kasparian A., Toledo D., Variations of Hodge structure of maximal dimension, Duke Math. J. 58 (1989), 669-694.

[9] Carlson J.A., Simpson C., Shimura varieties of weight two Hodge structures, in Hodge Theory (Sant Cugat, 1985), Lecture Notes in Math., Vol. 1246, Springer, Berlin, 1987, 1-15.

[10] Carlson J.A., Toledo D., Generic integral manifolds for weight-two period domains, Trans. Amer. Math. Soc. 356 (2004), 2241-2249, math.AG/0501078.

[11] Carlson J.A., Toledo D., Variations of Hodge structure, Legendre submanifolds and accessibility, Trans. Amer. Math. Soc. 311 (1989), 391-411. 
[12] Carlson J.A., Müller-Stach S., Peters C., Period mappings and period domains, Cambridge Studies in Advanced Mathematics, Vol. 85, Cambridge University Press, Cambridge, 2003.

[13] Donagi R., Generic Torelli for projective hypersurfaces, Compositio Math. 50 (1983), 325-353.

[14] Green M., Koszul cohomology and the geometry of projective varieties. II, J. Differential Geom. 20 (1984), 279-289.

[15] Green M., Griffiths P., Algebraic cycles and singularities of normal functions. II, in Inspired by S.S. Chern, Nankai Tracts Math., Vol. 11, World Sci. Publ., Hackensack, NJ, 2006, 2006, 179-268.

[16] Griffiths P., Hermitian differential geometry and the theory of positive and ample holomorphic vector bundles, J. Math. Mech. 14 (1965), 117-140.

[17] Ivey T.A., Landsberg J.M., Cartan for beginners: differential geometry via moving frames and exterior differential systems, Graduate Studies in Mathematics, Vol. 61, American Mathematical Society, Providence, RI, 2003.

[18] Mayer R., Coupled contact systems and rigidity of maximal dimensional variations of Hodge structure, Trans. Amer. Math. Soc. 352 (2000), 2121-2144, alg-geom/9712001.

[19] Otwinowska A., Composantes de petite codimension du lieu de Noether-Lefschetz: un argument asymptotique en faveur de la conjecture de Hodge pour les hypersurfaces, J. Algebraic Geom. 12 (2003), 307-320.

[20] Otwinowska A., Composantes de dimension maximale d'un analogue du lieu de Noether-Lefschetz, Compositio Math. 131 (2002), 31-50.

[21] Voisin C., Hodge loci and absolute Hodge classes, Compositio Math. 143 (2007), 945-958, math.AG/0605766. 